University of Tennessee Health Science Center

UTHSC Digital Commons

\title{
Structural and Kinetics Studies of the Enzyme Dihydropteroate Synthase and the Implications for Antibiotic Resistance
}

\author{
Katherine A. Ayers \\ University of Tennessee Health Science Center
}

Follow this and additional works at: https://dc.uthsc.edu/dissertations

Part of the Medical Biochemistry Commons, and the Medical Cell Biology Commons

\section{Recommended Citation}

Ayers, Katherine A. , "Structural and Kinetics Studies of the Enzyme Dihydropteroate Synthase and the Implications for Antibiotic Resistance" (2009). Theses and Dissertations (ETD). Paper 333.

http://dx.doi.org/10.21007/etd.cghs.2009.0020.

This Thesis is brought to you for free and open access by the College of Graduate Health Sciences at UTHSC Digital Commons. It has been accepted for inclusion in Theses and Dissertations (ETD) by an authorized administrator of UTHSC Digital Commons. For more information, please contact jwelch30@uthsc.edu. 


\title{
Structural and Kinetics Studies of the Enzyme Dihydropteroate Synthase and the Implications for Antibiotic Resistance
}

\begin{abstract}
The goal of this study is to develop small molecule inhibitors of DHPS for the treatment of a variety of infectious diseases. According to the World Health Organization (WHO), infectious diseases kill more than 13 million people worldwide every year making it the second leading cause of death behind cardiovascular disease. The sulfonamide class of drugs has been in use since the 1930's to treat many infectious agents and act by targeting the enzyme dihydropteroate synthase (DHPS) of the prokaryotic and lower eukaryotic folate pathway.

DHPS is an ideal drug target because humans do not synthesize folate de novo and is a well validated system. However, the emergence of bacterial resistance has limited the efficacy of sulfonamides, and an increasing trend in drug resistance has heightened the need for development of new therapeutics. Although drug resistance has severely limited the clinical use of sulfonamides, the folate pathway, and DHPS in particular, remain an ideal target for therapeutic development. This is due to the fact that DHPS accommodates two substrates, para-aminobenzoic acid (pABA) and 6-hydroxymethyl-7,8-dihydropterinpyrophosphate (DHPPP), which bind to separate and distinct regions of the protein. Sulfonamides act by binding the $P A B A$ pocket composed largely of dynamic loop regions which fold in upon substrate binding to complete the active site. In contrast, the pterin pocket is a highly conserved, rigid binding site, and is predicted to be more suitable for the development of small molecule inhibitors. Therefore, we propose targeting the pterin binding site as a means of developing novel small molecule inhibitors of DHPS.
\end{abstract}

The advantages of this method are that pterin binds to a distinct region of DHPS separate from the pABA binding site and removed from areas known to accommodate sulfonamide resistance. In addition, it provides a novel approach to exploit a well validated drug target. We hypothesize that pterin-based compounds will provide a new class of antibiotics that will overcome the problems of drug resistance and provide novel therapeutics for a broad spectrum of infectious diseases.

\section{Document Type}

Thesis

\section{Degree Name}

Master of Science (MS)

\section{Program}

Biomedical Sciences

\section{Research Advisor}

Stephen White, PhD

\section{Keywords}

Antibiotic Resistance, Dihydropteroate Synthase, Sulfamethoxazole, X-ray Crystallography

\section{Subject Categories}

Medical Biochemistry | Medical Cell Biology | Medical Sciences | Medicine and Health Sciences 
Structural and Kinetics Studies of the Enzyme Dihydropteroate Synthase and the Implications for Antibiotic Resistance

\author{
A Thesis \\ Presented for \\ The Graduate Studies Council \\ The University of Tennessee \\ Health Science Center
}

In Partial Fulfillment

Of the Requirements for the Degree

Master of Science

From The University of Tennessee

By

Katherine A. Ayers

May 2009 
Dedication

To my beloved husband, Clay. 


\section{Acknowledgements}

I would like to express my gratitude to everyone who has helped me in my endeavor of pursuing a Master of Science degree. To Dr. Stephen White, without his immense knowledge and hard work, this research project would not have been possible. To my committee members, Dr. Susan Senogles and Dr. Richard Lee for their advice and experience. To Tracey Lee and Dr. Darcie Miller, as well as the other members of the White lab, for their constant care and mentorship. Lastly, I must thank my family for their love and support. 


\begin{abstract}
The goal of this study is to develop small molecule inhibitors of DHPS for the treatment of a variety of infectious diseases. According to the World Health Organization (WHO), infectious diseases kill more than 13 million people worldwide every year making it the second leading cause of death behind cardiovascular disease. The sulfonamide class of drugs has been in use since the 1930's to treat many infectious agents and act by targeting the enzyme dihydropteroate synthase (DHPS) of the prokaryotic and lower eukaryotic folate pathway.

DHPS is an ideal drug target because humans do not synthesize folate de novo and is a well validated system. However, the emergence of bacterial resistance has limited the efficacy of sulfonamides, and an increasing trend in drug resistance has heightened the need for development of new therapeutics. Although drug resistance has severely limited the clinical use of sulfonamides, the folate pathway, and DHPS in particular, remain an ideal target for therapeutic development. This is due to the fact that DHPS accommodates two substrates, para-aminobenzoic acid (pABA) and 6hydroxymethyl-7,8-dihydropterin-pyrophosphate (DHPPP), which bind to separate and distinct regions of the protein. Sulfonamides act by binding the $p$ ABA pocket composed largely of dynamic loop regions which fold in upon substrate binding to complete the active site. In contrast, the pterin pocket is a highly conserved, rigid binding site, and is predicted to be more suitable for the development of small molecule inhibitors. Therefore, we propose targeting the pterin binding site as a means of developing novel small molecule inhibitors of DHPS.
\end{abstract}

The advantages of this method are that pterin binds to a distinct region of DHPS separate from the $p$ ABA binding site and removed from areas known to accommodate sulfonamide resistance. In addition, it provides a novel approach to exploit a well validated drug target. We hypothesize that pterin-based compounds will provide a new class of antibiotics that will overcome the problems of drug resistance and provide novel therapeutics for a broad spectrum of infectious diseases. 


\section{Table of Contents}

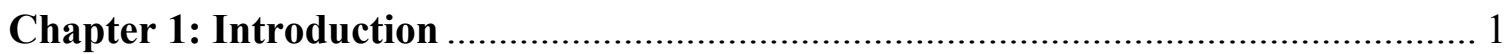

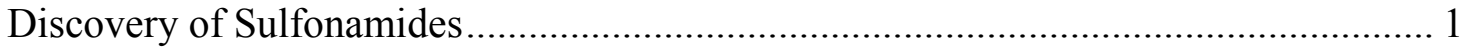

Sulfonamides and the Treatment of Infectious Diseases ............................................... 2

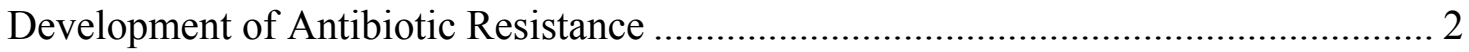

DHPS and the Folate Biosynthetic Pathway.............................................................. 4

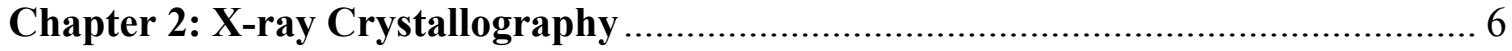

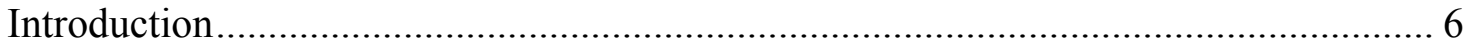

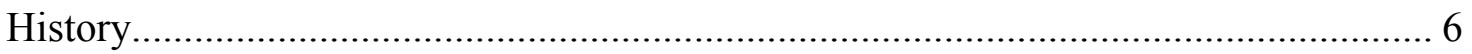

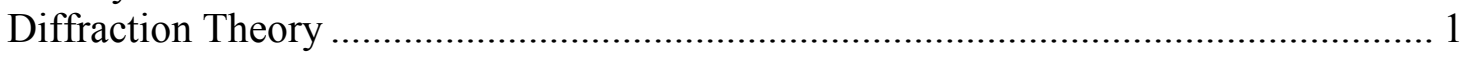

Chapter 3: Structural Studies of the Dihydropteroate Synthase (DHPS) ................ 10

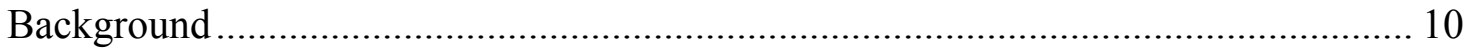

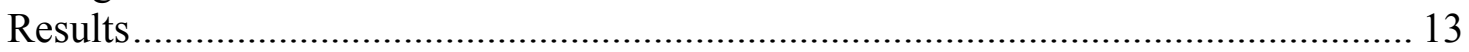

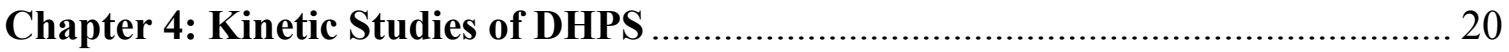

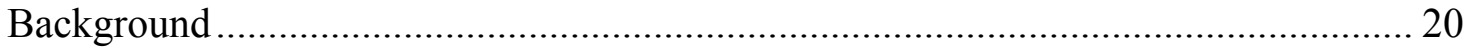

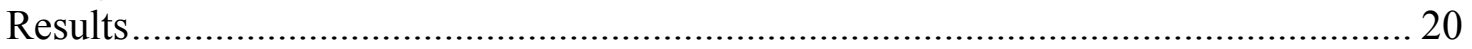

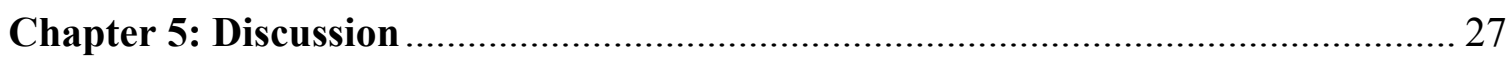

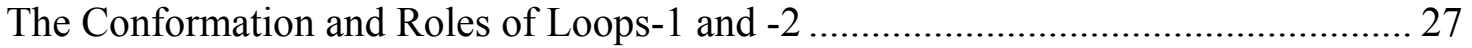

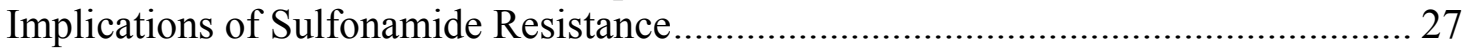

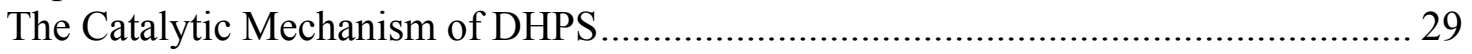

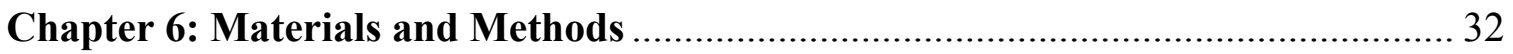

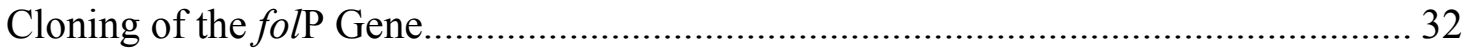

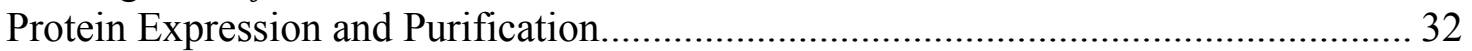

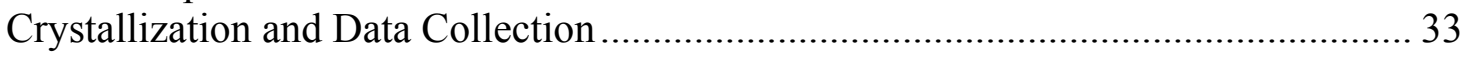

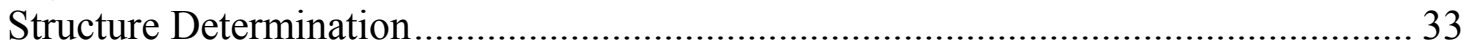

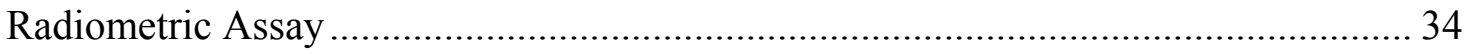

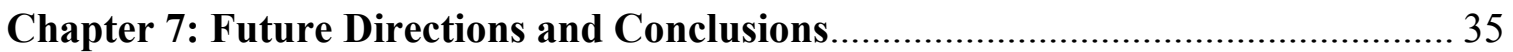

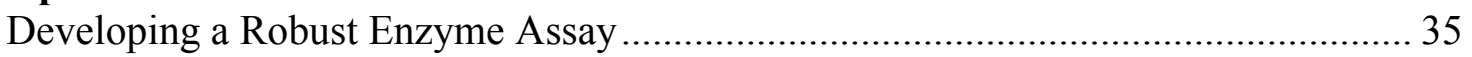

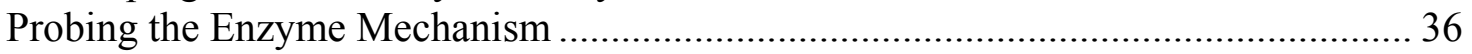

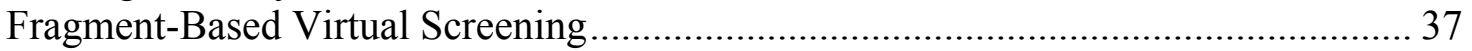

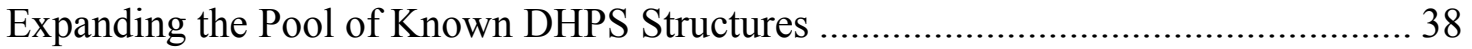

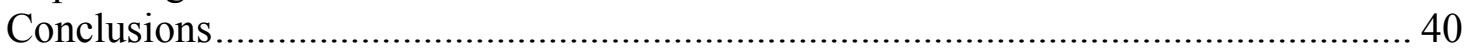

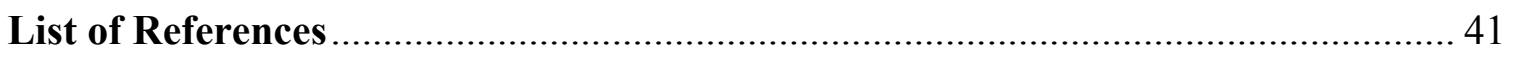

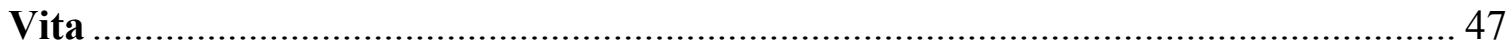




\section{List of Tables}

Table 1.1: Types of Sulfonamides and Therapeutic Uses........................................2

Table 1.2: Resistance Mutations from Clinical Isolates ........................................

Table 3.1: Crystallization Data of the DHPS Double Mutant Construct.....................18

Table 4.1: Kinetics Study of Mutant Alleles .........................................................22

Table 5.1: Sidechains Involved in Substrate Binding .............................................30 


\section{List of Figures}

Figure 1.1: Structure of Sulfonamides.................................................................

Figure 1.2: Schematic Representation of Tetrahydrofolate Synthesis ........................5

Figure 3.1: Structure of the DHPS Active Site...................................................... 11

Figure 3.2: Schematic Representation of the DHPS Transition State .........................11

Figure 3.3: DHPS Sequence Alignment ....................................................... 12

Figure 3.4: Crystal Packing Interactions Involving Loop-1 .................................14

Figure 3.5: Crystal Packing Interactions Involving Loop-2 ...................................15

Figure 3.6: Analysis of the WT B. anthracis Crystal Packing .................................16

Figure 3.7: Crystals of the $B$. anthracis DHPS ......................................................17

Figure 3.8: Active Site with Functional Conformation of Loop-2 ...........................17

Figure 4.1: Results from the ELIPA Experiment ..........................................22

Figure 4.2: Results from the Radiometric Assay ...............................................23

Figure 4.3: F33L Sequence Verification .....................................................24

Figure 4.4: Crystallization of the P69S, T67A, and F33L Mutant Alleles.................25

Figure 5.1: Schematic Representation of the Formation of Antibiotic Resistant

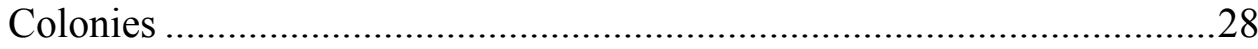

Figure 7.1: Purification Gels of $Y$. pestis and F. tularensis DHPS ...........................39 


\section{Chapter 1: Introduction}

\section{Discovery of Sulfonamides}

Sulfonamides were the first antimicrobial to be developed and their discovery laid the foundation for many of the concepts and techniques used in modern drug discovery. They were first discovered in 1932 by German scientist Gerhard Domagk. Working at the I. G. Farbenindustrie on methods for staining pathogenic bacteria, Domagk observed that an azo dye, Prontosil Rubrum, protected mice and rabbits against lethal doses of staphylococci and streptococci, but was ineffective in cell cultures (Domogk, 1935). It was later shown that the azo linkage of Prontosil Rubrum is metabolized by liver enzymes to yield the active drug, sulfanilamide, and developed the concept of bioactivation (Domogk, 1935) (Figure 1.1A). During the 1940's, it was shown that addition of para-aminobenzoic acid ( $p \mathrm{ABA}$ ) to bacteria colonies could overcome drug activity indicating that sulfonamides target the folate biosynthetic pathway (Brown, 1962). However, it was nearly three decades before Richey and Brown (Richey \& Brown, 1969) demonstrated that sulfanilamide was a potent inhibitor of the folate enzyme dihydropteroate synthase (DHPS) by purifying DHPS and performing in vitro analysis (Brown, 1962).

DHPS catalyzes the condensation of para-aminobenzoic acid (pABA) and 6hydroxymethyl-7,8-dihydropterin-pyrophosphate (DHPPP) to form dihydropteroate, a precursor of tetrahydrofolate which is used for DNA synthesis. The sulfonamide group of sulfanilamide mimics the carboxyl functional group of $p A B A$. Since sulfur is a more electron rich element than carbon, the $\mathrm{pKa}$ of the molecule is increased to 10.4 as compared to the $p \mathrm{ABA} \mathrm{pKa}$ of $\sim 6.5$. Therefore, second generation sulfonamides were optimized with the addition of an electron withdrawing ring to lower the $\mathrm{pKa}$ making them more efficient inhibitors of DHPS (Figure 1.1B).

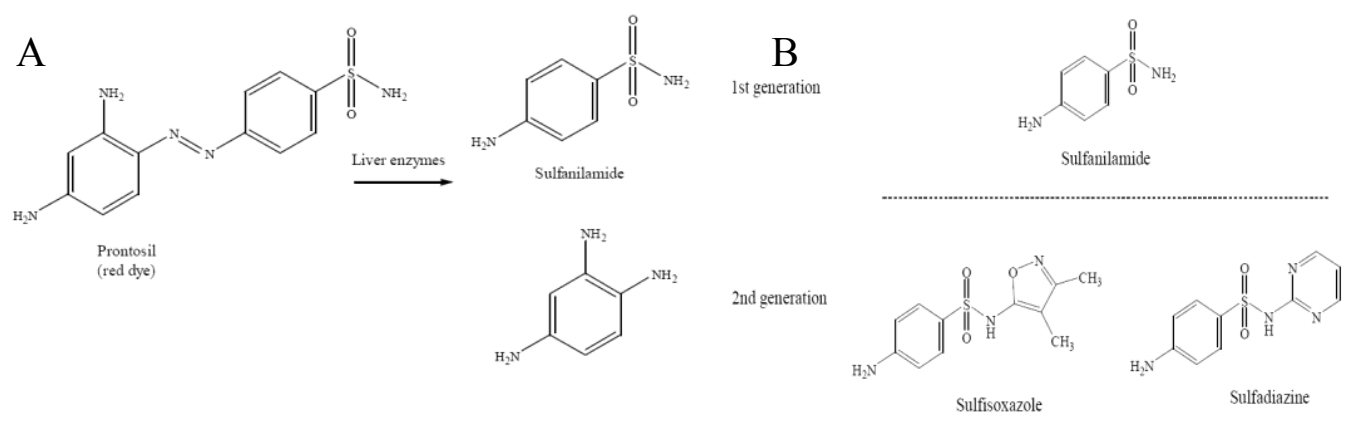

Figure 1.1: Structure of Sulfonamides. A. Schematic representation of prontosil metabolism by liver enzymes. B. Structure of the first and second generation sulfonamides. 


\section{Sulfonamides and the Treatment of Infectious Diseases}

After their discovery in the early 1930's, sulfa drugs became widely used and served as a first line treatment against bacterial infections. Pharmaceutical companies aggressively researched sulfonamides at the outbreak of the Second World War to produce potent inhibitors of infectious agents to prevent wound infection. Prisoners at the Ravensbrueck concentration camp were used as test subjects by the German army to investigate the effectiveness of sulfonamides in preventing infections in open wounds (U.S. Holocaust Memorial Museum). As a result of these studies, soldiers were issued first aid kits containing sulfa powder which they were told apply to any open wounds incurred on the battlefield.

Sulfa powder, also known as co-trimoxazole, is a combination of sulfamethoxazole (SMX) and trimethoprim (TMP). TMP targets the enzyme dihydrofolate reductase (DHFR) which catalyzes the reduction of dihydrofolate to tetrahydrofolate in the folate pathway downstream of DHPS. Currently, sulfonamides are still an effect treatment for a variety of infectious disease (Table 1.1), but the increase in drug resistance has limited their clinical use.

\section{Development of Antibiotic Resistance}

Historically, sulfonamides have been used as first line treatments for several infectious diseases including malaria, leprosy, pneumonia, E. coli, and S. aureus. However, antibiotic resistance has emerged as an important factor that severely limits the clinical use of sulfonamides (Sköld, 2000; 2001), and resistance mutations in clinical isolates of many pathogenic organisms have been characterized (Table 1.2). The most common mechanism of acquired resistance has been linked to mutations in the folP gene which encodes for DHPS. The majority of these mutations are localized in Loops-1 and 2 which fold in upon substrate binding to complete the $p \mathrm{ABA} /$ sulfonamide binding site, and primarily affect inhibitor binding. Sulfonamide resistance does not always involve a

Table 1.1: Types of Sulfonamides and Therapeutic Uses.

\begin{tabular}{|c|c|}
\hline Sulfonamides & Therapeutic Indications \\
\hline \multicolumn{2}{|c|}{ trimethoprim-sulfamethoxazole Treatment and prophalaxis of pneumonia } \\
\hline & First attack of urinary infection \\
\hline pyrimethamine-sulfadiazine & Treatment and prophalaxis of cerebral toxoplasmosis \\
\hline silver sulfacetamine & $\begin{array}{l}\text { Prevention/ treatment of bacterial infection for burn } \\
\text { patients }\end{array}$ \\
\hline sodium sulfacetamie & Conjunctivitis and related superficial ocular infections \\
\hline $\begin{array}{l}\text { sulfadoxine and sulfalene in } \\
\text { combination with quinine }\end{array}$ & Chloroquine-resistant malaria \\
\hline
\end{tabular}


Table 1.2: Resistance Mutations from Clinical Isolates.

\begin{tabular}{|c|c|c|c|c|}
\hline Organisms & Mutation Observed & Corresponding $B a$ Residue & Structure & References \\
\hline E. coli & Phe28Lue, Ile & Phe33 & Loop 1 & Dallas et al., 1992; Fermer \& Swedberg, 1997 \\
\hline N. meningitides & Phe31Leu & Phe33 & Loop 1 & Fermer \& Swedberg et al., 1997 \\
\hline P. cariii & Phe23Leu & Phe33 & Loop 1 & Lane et al., 1997 \\
\hline M. leprea & Thr53Ile, Ala & Thr67 & Loop 2 & Kai et al., 1999 \\
\hline P. carinii & Thr55Ala & Thr67 & Loop 2 & $\begin{array}{l}\text { Lane et al., 1997; Kazanjian et al., 1998; Mei et } \\
\text { al., 1998; Wang et al., } 1997\end{array}$ \\
\hline P. falciparum & Ser436Ala, Phe & Thr67 & Loop 2 & Wang et al., 1997 \\
\hline S. pneumoniae & Arg58-Pro59 duplication & Arg68-Pro69 & Loop 2 & Kai et al., 1999 \\
\hline M. leprea & Pro55Leu & Pro69 & Loop 2 & Lane et al., 1997; Kazanjian et al., 1998 \\
\hline P. carinii & Pro57Ser & Pro69 & Loop 2 & Mei et al., 1998 \\
\hline E. coli & Pro64Ser & Pro69 & Loop 2 & Vedantam et al., 1998 \\
\hline S. pneumoniae & Arg Insertion after Gly60 & Gly 70 & Loop 2 & Padaycahee \& Klugman, 1999 \\
\hline S. pneumoniae & Ser61 duplication & Phe71 & Loop 2 & Maskell et al., 1997 \\
\hline P. carinii & His60Asp & Ala72 & Loop 2 & Lane et al., 1997 \\
\hline S. pneumoniae & Ile66-Glu67 duplication & Val74-Ser75 & Loop 2 & Lopez et al., 1987 \\
\hline P. carinii & Ile111Thr & Ile122 & Loop 4 & Lane et al., 1997 \\
\hline P. falciparum & $\begin{array}{l}\text { Lys540Glu } \\
\text { Gly194Cys, Ser193- }\end{array}$ & Asn 147 & Loop 5 & Wang et al., 1997 \\
\hline N. meningitides & $\begin{array}{l}\text { Gly194 } \\
\text { Duplication }\end{array}$ & Gly188 & Loop 6 & $\begin{array}{l}\text { Fermer et al., 1995; Fermer \& Swedberg et al., } \\
1997\end{array}$ \\
\hline P. falciparum & Ala581Gly & Gly190 & Loop 6 & Wang et al., 1997 \\
\hline P. falciparum & Ala613Ser, Thr & Gly224 & $\alpha 7^{\prime}$ & Wang et al., 1997 \\
\hline P. carinii & Val248Gly & Ile246 & $\alpha 7^{\prime}$ & Lane et al., 1997 \\
\hline
\end{tabular}


single point mutation, but is often found to contain multiple alterations in the folP gene, resulting in changes of up to $10 \%$ of the amino acid sequence as compared to sensitive isolates (Radstom et al., 1992; Quarnstrom \& Swedberg, 2000).

\section{DHPS and the Folate Biosynthetic Pathway}

DHPS is a key enzyme in the folate biosynthetic pathway which catalyzes the condensation of 6-hydroxymethyl-7,8-dihydropterin-pyrophosphate (DHPPP) with paraaminobenzoic acid ( $p \mathrm{ABA})$ in the production of the intermediate dihydropteroate (Figure 1.2). The pathway serves to generate the cofactor tetrahydrofolate, which is essential to all organisms for the metabolic reactions that involve one-carbon transfer such as DNA synthesis. Prokaryotes and some lower eukaryotes require these enzymes for folate biosynthesis. However, higher eukaryotes utilize high affinity transport systems which absorb folate from the gut in the form of folic acid (Matherly, 2001). Therefore, DHPS is an ideal drug target because humans do not synthesize folate de novo and is a well validated system. Chapter 4 of this thesis deals with our efforts to structurally characterize the DHPS enzyme in complex with sulfonamide inhibitors to better understand the structural determinants of sulfonamide resistance. 


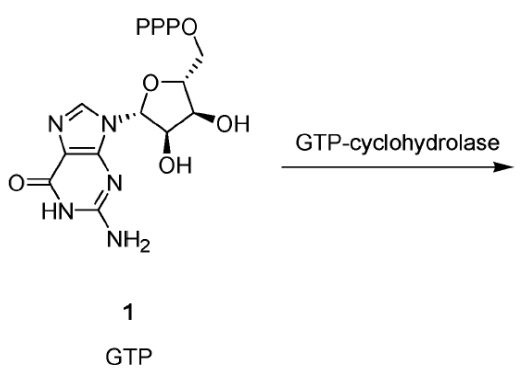

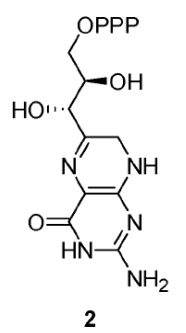

2

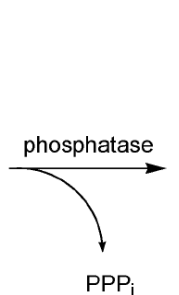

$\mathrm{PPP}_{\mathrm{i}}$

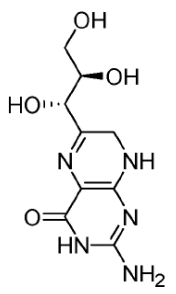

7,8-Dihydroneopterin
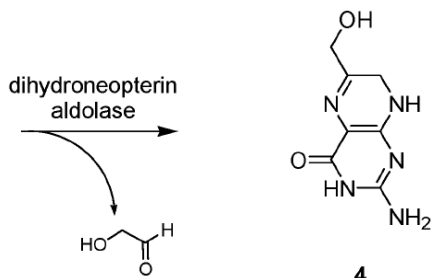

4

6-Hydroxymethyl-7,8 dihydropterin

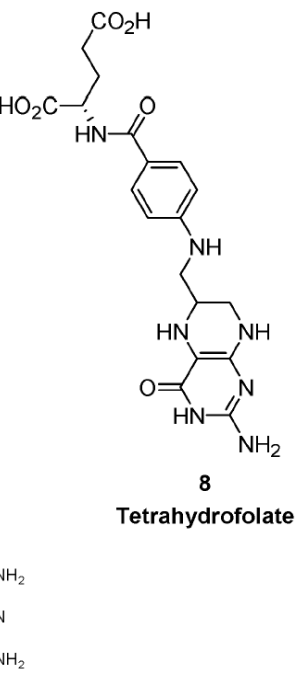

10

Figure 1.2: Schematic Representation of Tetrahydrofolate Synthesis. 


\section{Chapter 2: X-ray Crystallography}

\section{Introduction}

X-ray crystallography is a method of determining the special arrangement of atoms within a crystal lattice. Crystals described as regular arrays of atoms which are able to scatter X-ray wave, primarily through the atom's electrons. Using the angles and intensities of the scattered beams, a three-dimensional model of the electrons within the crystal can be obtained. From the electron density, the mean position of the atoms can be determined as well as their chemical bonds. For this reason, x-ray crystal structures are useful for shedding light on chemical interactions and processes and serve as a basis for designing pharmaceuticals against diseases. Indeed, modern applications of x-ray crystallography have led to the development of a new area of research known as structure-based drug design. Structure-based drug design is a technique which utilizes known molecular structures in conjunction with computational methods to allow a drug molecule to be constructed within the biomolecule using knowledge of its structure and the nature of its active site.

\section{History}

Crystals have been scientifically investigated since the $17^{\text {th }}$ century when Johannes Kepler hypothesized that the hexagonal symmetry of snowflakes was due to a regular packing of water molecules (Kepler, 1611). After the $18^{\text {th }}$ century, investigation by Nicolas Steno (Steno, 1669) and Rene Just Hauy (Hauy, 1784) led to the conclusion that crystals can be described by simple stacking patterns of "blocks" of the same shape and size. By the mid $19^{\text {th }}$ century, William Hallowes Miller was able to identify crystal faces by giving each face a unique label of three small integers known as the Miller indices. These studies led to the idea that crystals are a regular three-dimensional array of atoms and molecules in a single unit cell which is repeated indefinitely along three principle directions. By the conclusion of the $19^{\text {th }}$ century, a complete catalog of possible crystal symmetries had been determined and would later be validated by X-ray crystallography.

At the turn of the century, Wilhelm Conrad Rontgen discovered X-rays which were quickly determined to be waves of electromagnetic radiation. However, X-rays are composed of photons, introduced by Albert Einstein, and thus are not only waves of electromagnetic radiation but also exhibit particle-like properties (Einstein, 1905). The idea to use crystals as a gradient for X-rays arose a collaborative effort between Paul Peter Ewald and Max von Laue. The team exposed a sphalerite crystal to an X-ray beam and recorded the diffraction pattern onto a photographic plate. Once the plate was developed, it revealed a large number of well-defined spots arranged in a pattern of intersecting circles around the spot produced by the central beam (Ewald, 1962). 
From that point on X-ray crystallography boomed. The atomic structure of table salt became the first to be solved in 1914 and proved the existence of ionic compounds (Bragg, 1914; 1922). X-ray diffraction was a widely accepted technique in material science, chemistry, mineralogy and metallurgy. Discovery after discovery was being made to find new ways of utilizing and improving the principle methods of structure determination. Soon, organic and biological molecules were being crystallized and studied with the work of Dorothy Crowfoot Hodgkin as the premier crystallographer. In 1964, she was awarded the Nobel Prize in Chemistry for solving the structures of cholesterol, vitamin B12, and penicillin. In the late 1950's, the structure of sperm whale myoglobin was solved by Max Perutz and Sir John Cowerdry Kendrew making it the first protein structure to be determined via X-ray crystallography. Since then, thousands of proteins, nucleic acids, and other biological molecules have been determined using this technique.

\section{Diffraction Theory}

Understanding of how diffraction theory relates to crystal structure determination begins by studying the work of William Lawrence Bragg. Bragg proposed a model in which a given reflection is associated with a set of evenly spaced sheets running through the crystal, usually passing through the centers of the atoms of the crystal lattice. The orientation of a particular set of sheets is identified by its Miller indices $(h, k, l)$, and let their spacing be noted by a distance, $d$. Incoming X-rays are scattered in a mirror-like fashion from each plane. The X-rays scattered from adjacent planes will combine constructively when the angle $\theta$ between the plane and the X-ray results in a path-length difference that is an integer multiple $n$ of the X-ray wavelength $\lambda$. This concept is known as constructive interference and can be represented by the formula below.

$$
2 d \sin \theta=n \lambda
$$

Therefore, diffraction only occurs at so called Bragg's peaks. That is, for an ideal crystal, the electron density is perfectly periodic and the Fourier transform, $F(\mathrm{q})$, is zero except when $q$ belongs to the reciprocal lattice, that is, to a particular oscillation in the electron density in the direction in which q points. The reciprocal of the reciprocal lattice therefore is the original lattice of the crystal.

X-ray crystallography is used to determine the density of electrons $f(\mathrm{r})$ throughout a given crystal, where $r$ represents the three-dimensional position vector within the crystal. To do this, X-ray scattering is used to collect data about its Fourier transform, $F(\mathrm{q})$,

$$
f(\mathbf{r})=\int \frac{d \mathbf{q}}{(2 \pi)^{3}} F(\mathbf{q}) e^{i \mathbf{q} \cdot \mathbf{r}}
$$

where the integral is summed over all possible values of q. The three-dimensional real vector $\mathrm{q}$ represents a point in reciprocal space. Since the length of q corresponds to $2 \pi$ 
divided by the wavelength of the oscillation, the following formula for a Fourier transform can be used:

$$
F(\mathbf{q})=\int d \mathbf{r} f(\mathbf{r}) e^{-i \mathbf{q} \cdot \mathbf{r}}
$$

where the integral is summed over all possible values of the position vector $r$ within the crystal.

The Fourier transform $F(q)$ has two main components which must be accounted for experimentally in order to calculate the electron density of a crystal lattice: a magnitude $|F(\mathrm{q})|$ and a phase $\varphi(\mathrm{q})$ related by the equation,

$$
F(\mathbf{q})=|F(\mathbf{q})| e^{i \phi(\mathbf{q})}
$$

The intensities of the reflections observed in X-ray diffraction are synonymous with the magnitudes $|F(\mathrm{q})|$ but give no information regarding the phases $\varphi(\mathrm{q})$. To obtain the phases, full sets of reflections are collected with known alterations to the scattering, and combining the magnitudes and phases yields the full Fourier transform $F(\mathrm{q})$, which may be inverted to obtain the electron density $f(\mathrm{r})$. Several methods are available for determining phase information.

- Ab initio phasing, also known as direct methods - Although this method is most commonly used for small molecules, it been used successfully to solve the phase problems for small proteins. If the resolution of the data is better than $1.4 \AA$ $(140 \mathrm{pm})$, direct methods can be used to obtain phase information, by exploiting known phase relationships between certain groups of reflections.

- Molecular replacement - This method is often used if a structure exists of a related structure, it can be used as a search model in molecular replacement to determine the orientation and position of the molecules within the unit cell. The phases obtained this way can be used to generate an electron density maps.

- Anomalous X-ray scattering (MAD or SAD phasing) - the X-ray wavelength may be scanned past an absorption edge of an atom, which changes the scattering in a known way. By recording full sets of reflections at three different wavelengths (far below, far above and in the middle of the absorption edge) one can solve for the substructure of the anomalously diffracting atoms and thence the structure of the whole molecule. The most popular method of incorporating anomalous scattering atoms into proteins is to express the protein in a methionine auxotroph (a host incapable of synthesising methionine) in a media rich in Selenomethionine, which contains Selenium atoms. A MAD experiment can then be conducted around the absorption edge, which should then yield the position of any methionine residues within the protein, providing initial phases. 
- Heavy atom methods (i.e., MIR) - If electron-dense metal atoms can be introduced into the crystal, direct methods or Patterson-space methods can be used to determine their location and to obtain initial phases. Typically, heavy atoms may be introduced either by soaking the crystal in a heavy atom-containing solution, or by co-crystallization (growing the crystals in the presence of a heavy atom). As in MAD phasing, the changes in the scattering amplitudes can be interpreted to yield the phases. Although this is the original method by which protein crystal structures were solved, it has largely been superseded by MAD phasing with selenomethionine.

In addition, for every reflection corresponding to a point $\mathrm{q}$ in the reciprocal space, there is another reflection of equal intensity at the opposite point $-\mathrm{q}$. This opposite reflection is known as the Friedel pair of the original reflection. This symmetry results from the mathematical fact that the density of electrons $f(\mathrm{r})$ at a position $\mathrm{r}$ is always a real number. As noted above, $f(\mathrm{r})$ is the inverse transform of its Fourier transform $F(\mathrm{q})$; however, such an inverse transform is a complex number. To ensure that $f(\mathrm{r})$ is real, the Fourier transform $F(\mathrm{q})$ must be such that the Friedel pairs $F(-\mathrm{q})$ and $F(\mathrm{q})$ are complex conjugates of one another. Thus, $F(-\mathrm{q})$ has the same magnitude as $F(\mathrm{q})$ - that is, $|F|(\mathrm{q})=$ $|F|(-\mathrm{q})$ - but they have the opposite phase, i.e., $\varphi(\mathrm{q})=-\varphi(\mathrm{q})$ :

$$
F(-\mathbf{q})=|F(-\mathbf{q})| e^{i \phi(-\mathbf{q})}=F^{*}(\mathbf{q})=|F(\mathbf{q})| e^{-i \phi(\mathbf{q})}
$$

The equality of their magnitudes ensures that the Friedel pairs have the same intensity $|F|^{2}$. This symmetry allows one to measure the full Fourier transform from only half the reciprocal space. In crystals with significant symmetry, even more reflections may have the same intensity. In such cases, even less of the reciprocal space may need to be measured. 


\section{Chapter 3: Structural Studies of the Dihydropteroate Synthase (DHPS)}

\section{Background}

There are currently five known structures of DHPS: B. anthracis (Babaoglu et al., 2004), E. coli (Achari et al., 1997), S. aureus (Hampele et al., 1997), M. tuberculosis (Baca et al., 2000), and S. Pneumoniae (Levy et al., 2008) as well as the structure of the S. cerevisiae DHPS (Lawrence et al., 2005) which is fused to an additional enzyme of the folate pathway, 6-hydroxymethyl-7,8-dihydropterin pyrophosphokinase (HPPK). The DHPS enzyme is a classic $(\alpha / \beta)_{8}$ TIM barrel. Several structures of DHPS in complex with 6-hydroxymethyl-pterine-pyrophosphate (PtPP) and 6-hydroxymethyl-pterinmonophosphate (PtP) have been reported (Hampele et al., 1997; Baca et al., 2000; Babaoglu et al., 2004; Levy et al., 2008) and the pterin binding site is well characterized (Figure 3.1A,B). In addition, the structure of DHPS complexed with pteroic acid, a product analog, has been reported (Babaoglu et al., 2004) which revealed for the first time the position of the $p$ ABA binding site (Figure 3.1C). These structures build a preliminary model of the DHPS active site in which pterin binds into a highly conserved, rigid pocket buried deep within the DHPS enzyme (Figure 3.1D). In contrast, $p$ ABA binds to a surface region of the enzyme composed mainly of flexible loop regions which stabilize upon substrate binding to complete the active site. Based on the PtPP and PtP structures, Baca et al proposed a SN2 transition-state shown in Figure 3.2. In addition, it has been shown that substrate binding into the DHPS active site proceeds via an ordered mechanism where $p \mathrm{ABA}$ binding is dependent upon the presence of bound DHPPP (Babaoglu et al., 2004; Levy et al., 2008). Levy et al. demonstrated that pyrophosphate was efficient to facilitate $p \mathrm{ABA}$ binding in the absence of a pterin moiety correlating to a SN2 mechanism.

These structural studies show that the DHPS active site is divided into two separate, distinct regions: the pABA binding site and the pterin binding site. The pterin binding site is a highly conserved pocket embedded within the core of the DHPS structure and is composed of four key interactions with surrounding residues (Figure 3.1A,B,C): Arg254 pi-stacking interactions, Asn $120 \mathrm{H}$-acceptor, Asp184 H-acceptor, and Lys $220 \mathrm{H}$-donor. Upon pterin binding, Lys220 extends to form a platform for $p \mathrm{ABA}$ to bind as indicated in the pteroic acid structure (Figure 3.1C). $p$ ABA is also stabilized in the active site by an H-bonding interaction with Ser221. In addition, Levy et al. demonstrated that Loops- 1 and -2 fold in upon pterin binding to complete the $p \mathrm{ABA}$ binding site. However, these loop interactions have yet to be structurally characterized. Since the pterin binding pocket is highly conserved (Figure 3.3), it seems logical that it would be more difficult for the enzyme to accommodate mutations against pterin-based compounds. In addition, mutations that could potentially cause resistance to pterin-based inhibitors are likely to disrupt structural integrity since the pterin binding site is buried within the protein. In contrast, the $p$ ABA binding site involves flexible loop regions near the surface of the protein, and the rapid emergence of multiple sulfonamide resistance mutations clearly show that they are easily tolerated within the overall structure of the 


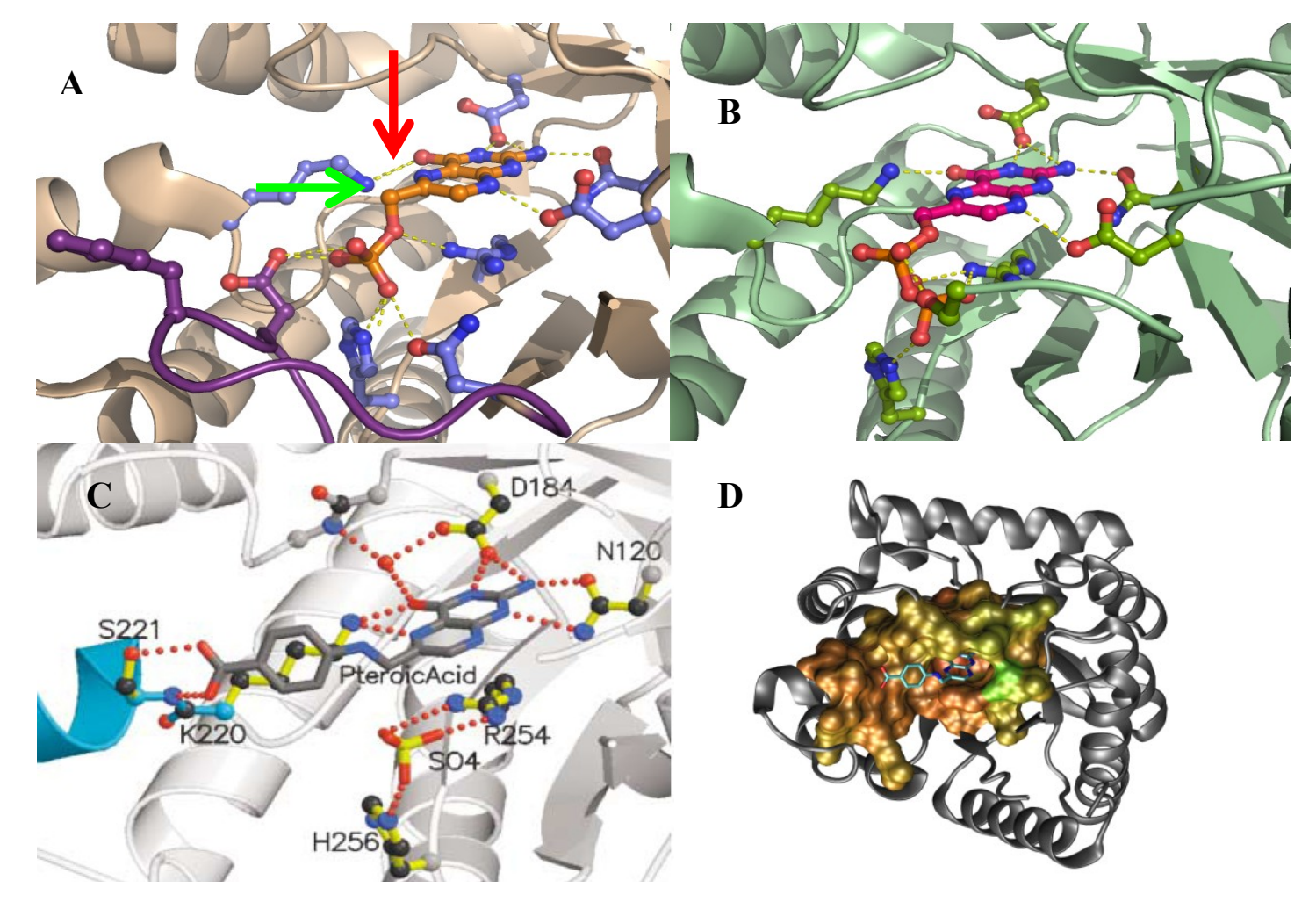

Figure 3.1: Structure of the DHPS Active Site. A. DHPS active site with bound PtP (PDB: 1EYE). Red arrow shows the direction of nucleophilic attack proposed by Baca et $a l$. and the green arrow represents the direction of nucleophilic attack indicated by the pteroic acid structure. B. DHPS active site with bound PtPP (PDB: 1TWS). C. DHPS active site with bound pteroic acid. Compound source of structure: Babaoglu, K., Qi, J., Lee, R.E. and White, S.W. (2004). Crystal structure of 7,8-dihydropteroate synthase from Bacillus anthracis : mechanism and novel inhibitor design. Structure, 12 :1705-1717. D. Surface representation of DHPS active site with bound pteroic acid indicating the separate binding pockets for each substrate. Note that the pterin moiety is deeply imbedded within the protein structure.

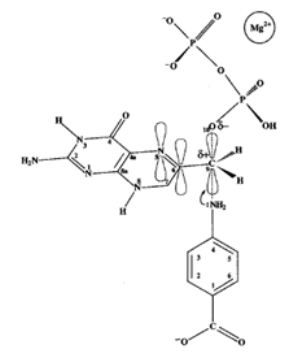

Figure 3.2: Schematic Representation of the DHPS Transition State. 
|S. aureus|

| E. coli |

|Y.pestis|

|B. anthracis|

|M. tubercul osis|

|S. aureus|

|E. coli |

|Y.pestis|

| B. anthracis|

|M. tuberculasis|

|S. aureus|

|E. coli |

|Y.pestis|

| B. anthracis|

|M. tuberculosis|

| S. aureus|

|E. coli |

|Y.pestis|

| B. anthracis|

|M. tuberculosis|

S. aureus

|E. coli |

|Y.pestis|

| B. anthracis|

| M. tuberculosis|
MTKTKI MG ILNVTPDSFSDGGKFTNWET A INRVK HM IDEG AD ------MKLF AQGTSL D-LSHP HVMG ILNV TP DSFSDGGTHNSL ID AVKH ANLM IN AG AT ------MHLT ÄRGL TL D-LSRP ONMG ILNV TP DSFSDGGC HNNL DQÄLQH AQRMLS AG AT MKWD YD LRCGEY -- TLNLNE KTL IMG ILNVTP DSFSDGGS YNEVD A AVRH AKKEMRDEG AH MGVLNVTD DSFSDGGC YLDLDD AVKHGL MMAM BG BG

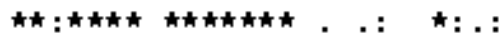

IIDVGGVSTRPGHE MVTLEE ELNRVL PVVE AIVG-F DVKISVDTFRSEVÄEACL KLGVDM IIDVGGESTRPG Ả EVSVEE ELQRVI PVVE A I AQRF EVWISVDTSKPEVIRESAKVGÄHI

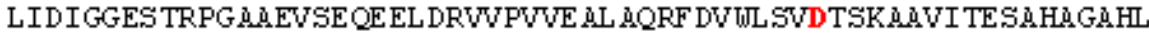

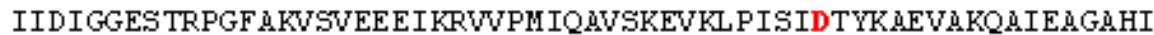
TVDVGGESSRPGATRVDP AVETSRVIPVVKEL AMQ-GITVSIDTMR ADVARA HLGNGAQM

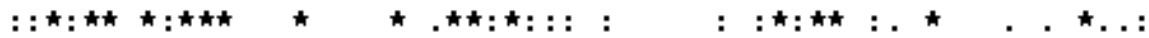

IRDQWAGL YD HRMF QIVA KY D AE I ILMHNGNGNRDE--------PVVE EMLTSLLÄQAHQ INDIRS-LSE PG ALE EA DAETGL PVCLMHMQGNPKTMQE AP -KYD DVF ẢEVNR YF IEQI AR INDIRS-LQE PG ALEA A. KKTGL PVCLMHMQGQPKNMOHSP-YYDDL MTD INRFF QHHIER IRD I WG AKA ÄE PK I A EV A A HYDVP I ILMHNRDNM--------NYRNL MADM IADL YDSI KI VHDVSGGR ADPAMGPLLAEADVPUVLMHURAVSADTPHVPVRYGNVVAEVRADLLASV AD

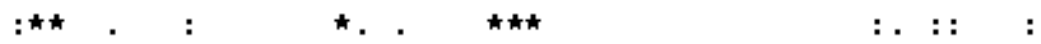

AK IA GI PSNK IWLD PG IGF AKTRNEE AEVMARLDEL VA TE YPVL LA TSRKRF TKEMMGYD CEQA GI AKEKLLLD PGFGFGKNLSHNYSLL \&RLAEF HHFNLPLLVGMS RKSM IGQLL--N CV A. GI AKNKLLLD PGFGFGKNL A HNYQLL AHLSEL HHFE LPLLVGMSRKSMVGQL L- -N AKD A GVRD EN I ILD PG IGF AKTPE QNLE AMRNLEQLNVLGYPVL LGTSRKSF IGHVL--D AV A. GVDP ARLVLD PGLGF AKT AQHNWA IL HALP ELVA TG IPVLVG ASRKRF LG ALL-A

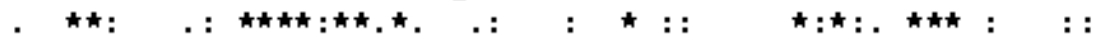

Figure 3.3: DHPS Sequence Alignment. The " $"$ means that the residues in that column are identical in all sequences in the alignment. The "." means that conserved substitutions have been observed. The "." means that semi-conserved substitutions have been observed. Residues known to interact with pterin moiety are in red. 
protein. It is a long term goal of this project to develop new inhibitors of DHPS which target the pterin binding site.

\section{Results}

While much of the DHPS active site has been structurally elucidated, key interactions involving Loops- 1 and -2 have yet to be determined. Therefore, it is the purposes of this study to construct a more desirable model by which to study these loop interactions. Analysis of the wild type B. anthracis structure revealed crystal packing interactions involving Loops-1 and -2. As shown in Figure 3.4, Loop-1 is engaged in an intramolecular packing interaction involving Phe33. Based on the M. tuberculosis structure (Baca et al., 2000), Loop-1 is a central component of the active site, and any movement of the loop would most likely destroy the crystals. Also, Phe33 has been shown to be a resistance mutation in drug resistant strains of $N$. meningitides and $E$. coli (Table 1.2). Loop-2, on the other hand, is folded into the pterin biding pocket forming $\pi$ stacking interactions with Arg254 (Figure 3.5). These interactions bind the loops into artificial conformations which prevent them from folding into the active site and, thereby, hindering a functional conformation of these loops in structural studies. Several residues located on Loop-2 have been shown to be involved in resistance mutations as well (Table 1.2). For these reasons, we have used site-directed mutagenesis techniques on surface residues to break crystal packing interactions in an attempt to grow a different crystal form in which these loops are free to interact with substrates upon binding. Analysis of the crystal lattice led to the following mutations: Tyr39His and His 137Glu (Figure 3.6). These mutations were made based on the $Y$. pestis sequence. Furthermore, biochemical studies using the radio-labeled assay (Materials and Methods) showed no significant change in catalytic activity (data not shown).

After several crystal trials, crystals were found in a $1.5 \mathrm{M}$ sodium malonate $\mathrm{pH}=$ 5.00 condition (Figure 3.7A). The outward appearances of these crystals were strikingly different from the wild type crystals grown by Babaoglu which were grown in a $1.3 \mathrm{M}$ $\mathrm{Li}_{2} \mathrm{SO}_{4}, 0.1 \mathrm{M}$ Bis-Tris propane, $\mathrm{pH} 9.0$ condition (Figure 3.7B) indicating that the mutations were successful in yielding an alternative crystal packing arrangement. Crystals of the DM construct were grown in space group P $2{ }_{1} 2_{1} 2_{1}$ with unit cell dimensions $\mathrm{a}=100.3 \AA, \mathrm{b}=107.9 \AA, \mathrm{c}=243.4 \AA$, and the structure was solved to $2.0 \AA$ resolution by molecular replacement using the wild type $B$. anthracis structure as a search model. Superposition of the double mutant structure showed no major conformational changes in the protein architecture (data not shown).

Presented here is the crystal structure of the double mutant (DM) B. anthracis DHPS (Figure 3.8). Analysis of the structures using PROCHECK (Laskowski et al., 1993) showed that each has excellent stereochemistry and final R-factors that are appropriate for their resolutions. Structure statistics are shown in Table 3.1. These structures are comparable to the native DHPS structures preciously determined. There are no significant differences apart from the conformation of loops and residues which compose the actives site regions. There is no electron density for the N-terminal 


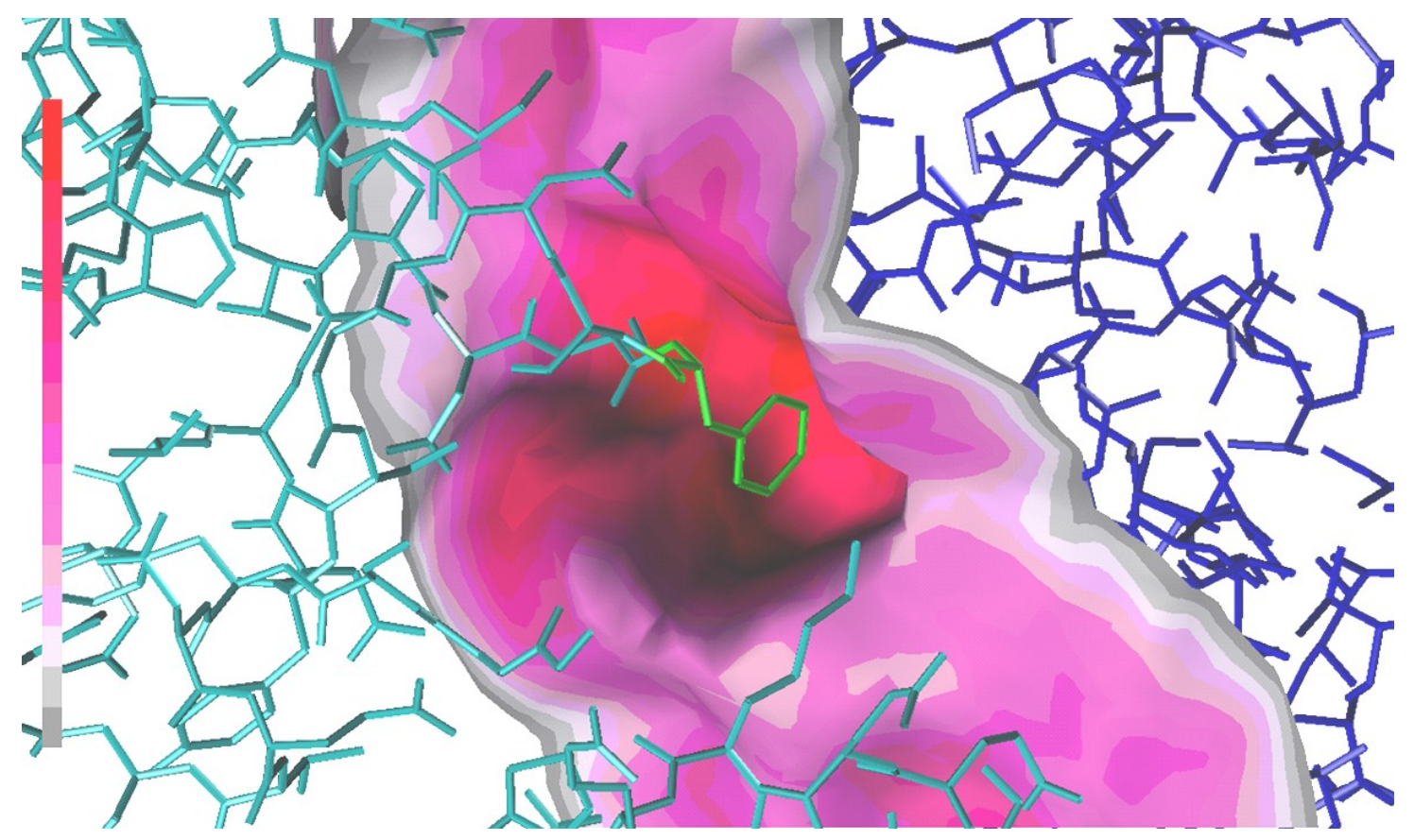

Figure 3.4: Crystal Packing Interactions Involving Loop-1. 

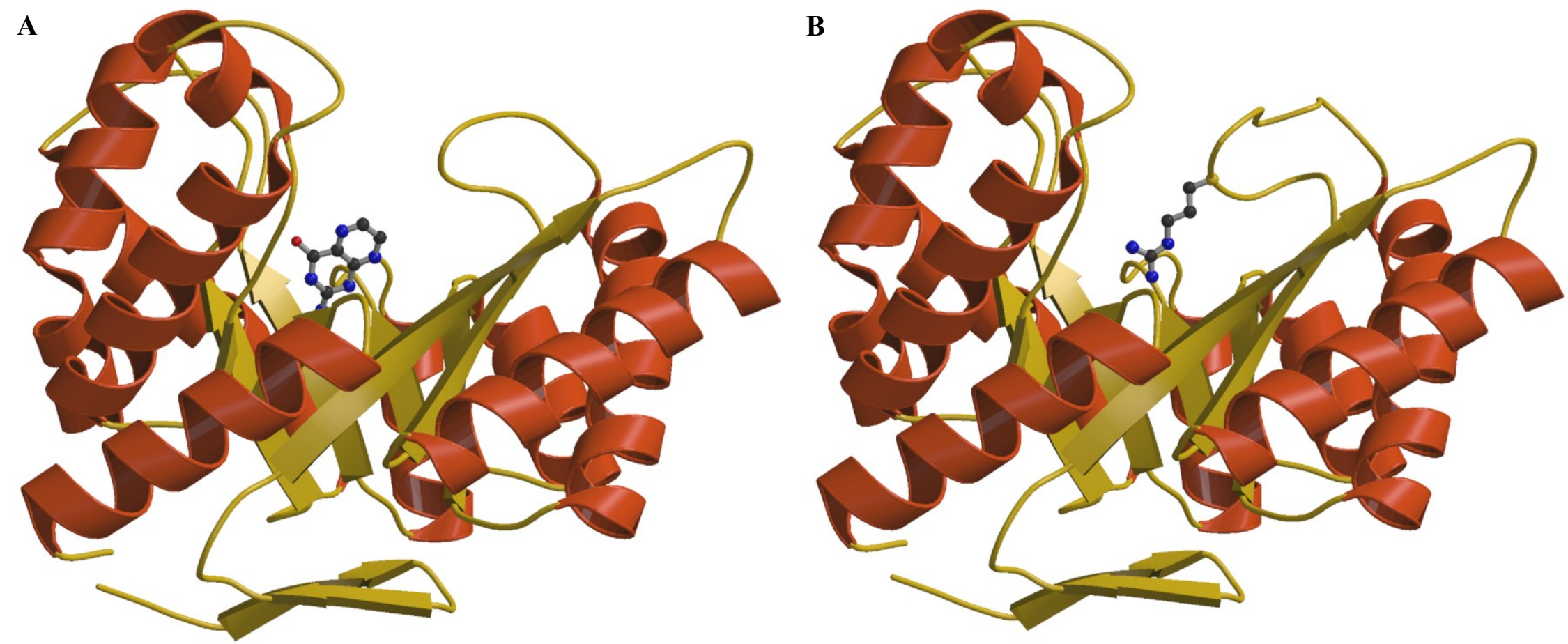

Figure 3.5: Crystal Packing Interactions Involving Loop-2. A. This structure shows pterin binding into the pterin-pocket. B. This structure shows Arg68 binding into the pterin-pocket. Compound source of structure: Babaoglu, K., Qi, J., Lee, R.E. and White, S.W. (2004). Crystal structure of 7,8-dihydropteroate synthase from Bacillus anthracis : mechanism and novel inhibitor design. Structure, $12: 1705-1717$. 

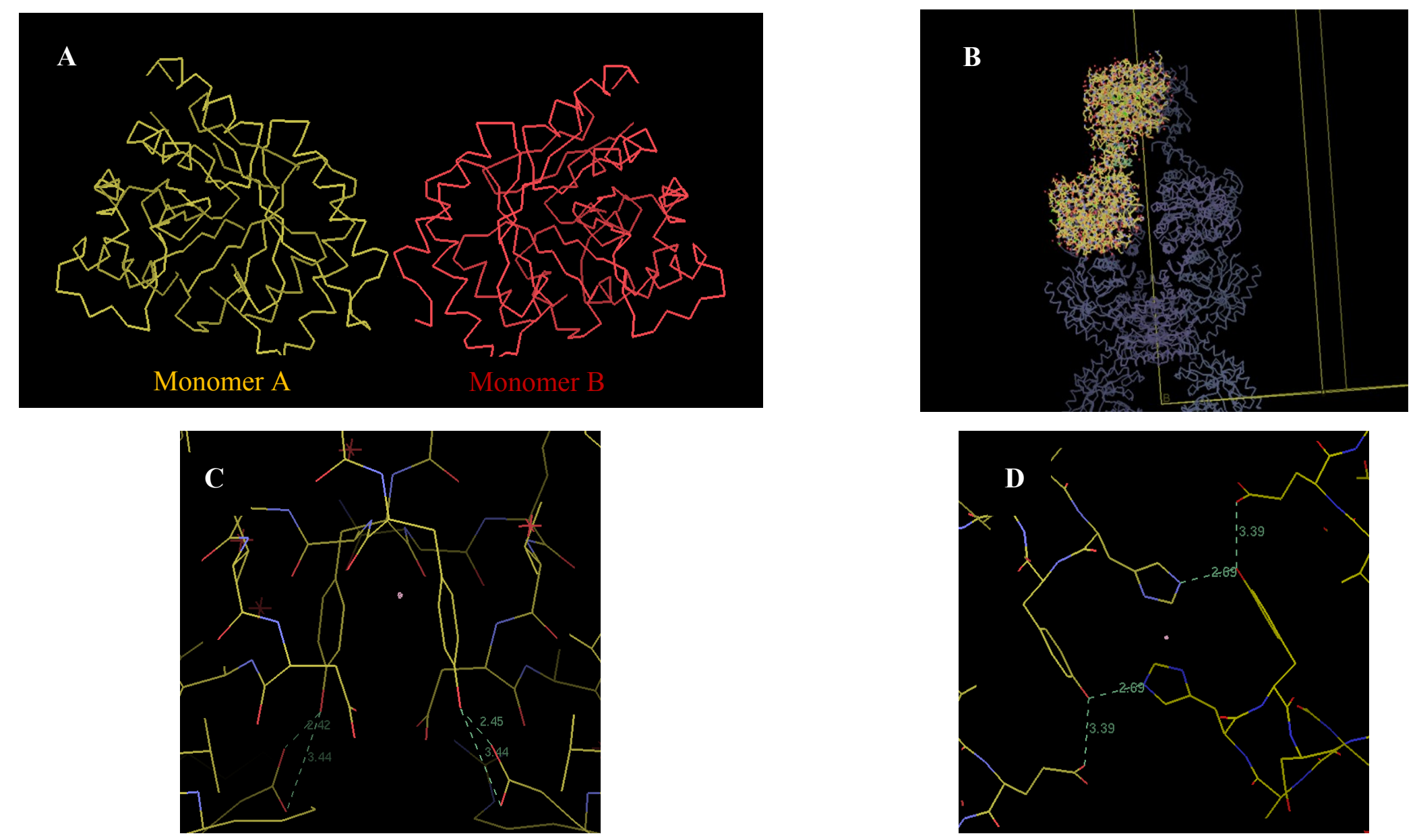

Figure 3.6: Analysis of WT B. anthracis Crystal Packing. A. Crystal packing interactions between monomers A and B. B. Crystal packing interactions between symmetry mates. C. Crystal packing interaction involving Tyr39. D. Crystal packing interaction involving His 137. 


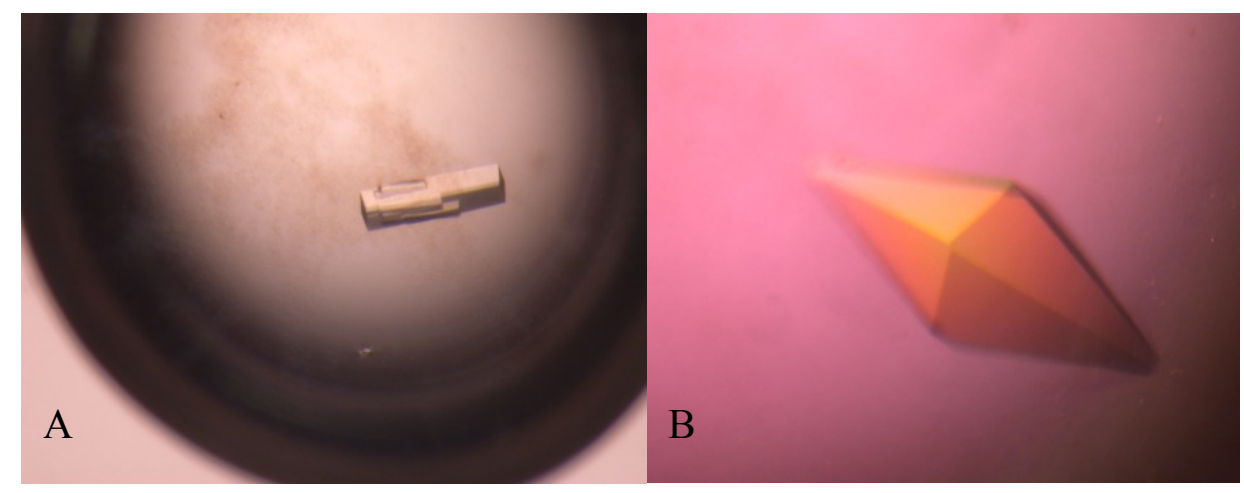

Figure 3.7: Crystals of the B. anthracis DHPS. A. Crystals of the double mutant construct. B. Crystals of WT B. anthracis.

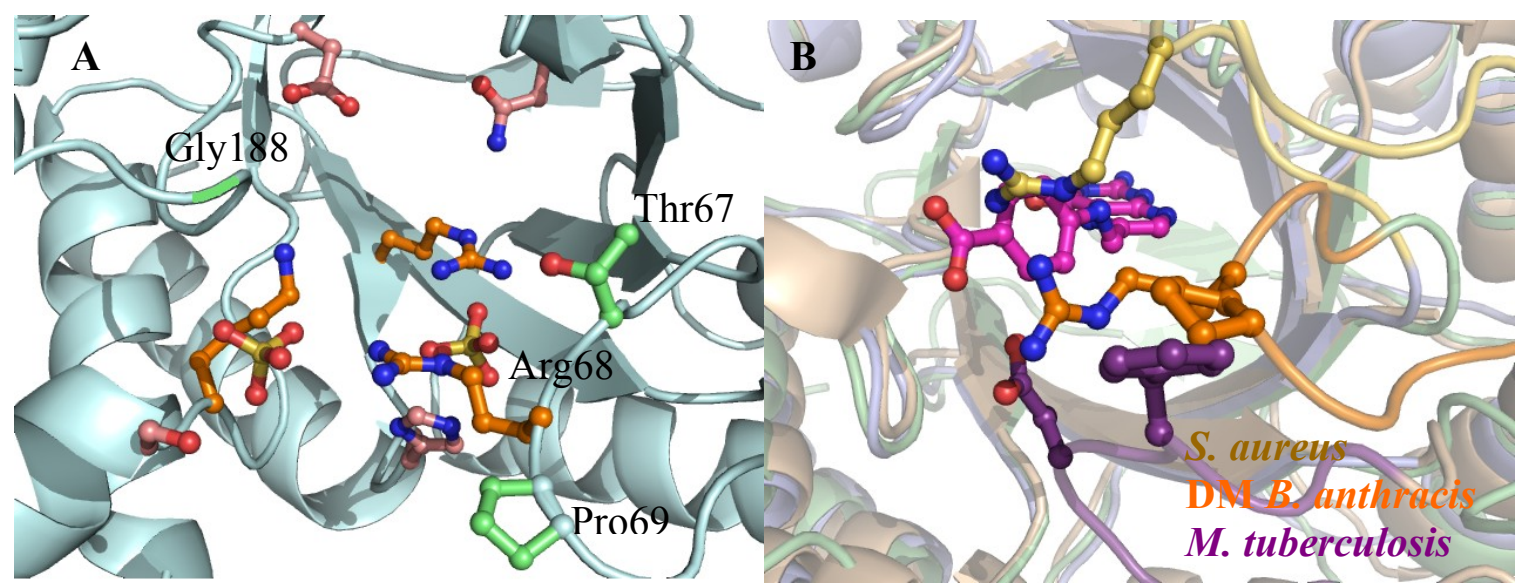

Figure 3.8: Active Site with Functional Conformation of Loop-2. A. This view shows stabilized Loop-2 with Arg68 directed towards the $p$ ABA binding site. Residues in green are involved in drug resistance. B. This view shows the DM Loop-2 (orange) with pteroic acid product analogue modeled in to the active site as well Loop-2 conformation from $S$. aureus (yellow) and Loop-1 conformation from M. tuberculosis (purple). 
Table 3.1: Crystallization Data of the DHPS Double Mutant Construct.

\begin{tabular}{ll}
\hline Data Collection & DM Construct \\
\hline Space Group & $\mathrm{P} 22_{1} 2_{1}$ \\
Cell Dimensions & \\
$\quad$ a,b,c $(\AA)$ & $100.357,107.999,243.443$ \\
$\quad \alpha, \beta, \gamma\left({ }^{\circ}\right)$ & $90,90,90$ \\
Resolution $(\AA)$ & $50-2.4$ \\
Rsym or Rmerge & $9.3(65.6)$ \\
I/ $\sigma \mathrm{I}$ & $12.2(1.5)$ \\
Completeness (\%) & $98.4(87.0)$ \\
Redundancy & $6.7(3.1)$ \\
Refinement Resolution $(\AA)$ & $50-2.6$ \\
No. Reflections & 113375 \\
Rwork/ Rfree & $20.1 / 25.6$ \\
No. Atoms & \\
$\quad$ Protein & 15912 \\
$\quad$ Ligand/ Ion & 120 \\
$\quad$ Water & 784 \\
Ramachandran Plot & \\
$\quad$ Most favored $(\%)$ & 88.4 \\
$\quad$ Additional allowed (\%) & 10.6 \\
Generously allowed $(\%)$ & 0.5 \\
Disallowed $(\%)$ & 0.5 \\
R.M.S. deviation & \\
Bond lengths $(\AA)$ & 0.010 \\
Bond angles $\left({ }^{\circ}\right)$ & 1.500 \\
\hline
\end{tabular}

Note: Values in parenthesis correlate to the highest resolution shell. 
methionine, the $\mathrm{N}$-terminal $6 \mathrm{xHis}$ tag, or the final three $\mathrm{C}$-terminal residues in any of the structures.

The overall fold of the $B$. anthracis DHPS has been described in previous works (Babaoglu et al., 2004). The basic fold of the protein is a classic $(\beta / \alpha)_{8}$ TIM barrel (Banner et al., 1975) in which repeating $\beta / \alpha$ units create an eight-stranded parallel $\beta$ barrel surrounded by eight $\alpha$-helices. In TIM barrel enzymes, the active site is located at the $\mathrm{C}$-terminal pole of the $\beta$-barrel and completed by interactions with surrounding, flexible loop regions. The same is true for DHPS, however, there is some debate as to the correct functional conformation of these loops as significant variations in conformation have been reported in the various orthologs.

Loop-1 (residues 29-37) is highly conserved, but is not fully visible in any of the eight monomers in the double mutant structure. Although it is highly conserved, its conformation is reportedly highly variable which indicates that Loop-1 is a highly mobile region of the DHPS active site. This would explain why Loop-1 is not visible in our structure as well as monomer B of the WT B. anthracis structure and one monomer of the $S$. aureus structure.

Loop-2 (residues 63-64) is also a highly conserved loop in the DHPS structure and is visible in three of the eight monomers of the asymmetric unit. It adopts a bent conformation (Figure 3.8A) in which R68 points towards the binding position of the carboxyl group of $p \mathrm{ABA}$. We believe that this conformation of Loop-2 is of functional significance and aids in stabilizing $p \mathrm{ABA}$ in the active site.

By modeling in the pteroic acid structure from the wild type $B$. anthracis complex (Babaouglu et al., 2000), we can visualize how this Loop-2 conformation may interact with the carboxylic acid of $p$ ABA. Furthermore, modeling in the various loop conformations from the M. tuberculosis and $S$. aureus structures sheds light on how these loops are able to move and assist one another to facilitate substrate binding (Figure 3.8B).

The double mutant construct also yields several implications into the mechanism of drug resistance. Specifically, the structure reveals the position of four key resistance mutation sites. First, it shows the position of Thr67 located on Loop-2 which is mutated to an Alanine in P. carinii and M. leprea mutant alleles (Figure 3.8A; Table 1.2). Second, the structure reveals the position of Pro69 also on Loop-2 (Figure 3.8A) which is mutated to a Serine in P. carinii and E. coli mutant alleles (Table 1.2) as well as the position of Arg68 on Loop-2. Furthermore, the apparent flexibility of Loop-2 suggests that Pro69 and Thr67 function to structurally stabilize Loop-2 in order to direct Arg68 towards the active site. Finally, the structure reveals the location Gly188 on Loop-6. Gly188 is mutated to Cysteine in N. meningitides drug resistant alleles (Table 1.2). The position of Gly188 suggests that the Gly188Cys mutation may function by sterically hindering the binding of sulfamethoxazole into the DHPS active site. 


\section{Chapter 4: Kinetic Studies of DHPS}

\section{Background}

Current structural studies (see Chapter 3) build a preliminary model of the DHPS active site in which pterin binds into a highly conserved, rigid pocket buried deep within the DHPS enzyme. In contrast, $p$ ABA binds to a surface region of the enzyme composed mainly of flexible loop regions which stabilize upon substrate binding to complete the active site. Based on the PtPP and PtP structures, Baca et al proposed a SN2 transitionstate. In addition, it has been shown that substrate binding into the DHPS active site proceeds via an ordered mechanism where $p \mathrm{ABA}$ binding is dependent upon the presence of bound DHPPP (Babaoglu et al., 2004; Levy et al., 2008). Levy et al. demonstrated that pyrophosphate was efficient to facilitate $p$ ABA binding in the absence of a pterin moiety correlating to a SN2 mechanism. However, the $p \mathrm{ABA}$ binding site predicted in the pteroic acid structure seemingly contradicts the SN2 mechanism as the direction of the nucleophilic attack is not optimal.

Understanding the mechanism of DHPS catalysis is particularly important for understanding the mode of sulfonamide resistance since sulfonamides inhibit the function of DHPS by competing with $p$ ABA for active site binding (Brown, 1962). However, in many instances these compounds undergo catalytic activity to produce sulfa-pterin adducts (Roland et al., 1979; Patel et al., 2004; Valderas et al., 2008) which have been implicated as inhibitors of dihydrofolate reductase (DHFR) (Patel et al., 2004). DHFR is an enzyme downstream of DHPS in the folate pathway and is the target for several inhibitors commonly used in synergism with sulfonamides. Historically, sulfonamides have been used as first line treatments for several infectious diseases including malaria, leprosy, pneumonia, E. coli, and S. aureus. However, drug resistance has emerged as an important factor that severely limits the clinical use of sulfonamides (Sköld, 2000; 2001), and resistance mutations in clinical isolates of many pathogenic organisms have been characterized. The majority of these mutations are localized in Loops- 1 and -2 which fold in upon substrate binding to complete the $p \mathrm{ABA} /$ sulfonamide binding site. For this purpose, we have developed a radiometric assay to understand the involvement of Loops1 and -2 in the development of sulfonamide resistance.

\section{Results}

We have developed two assays to be used for kinetic studies, ELIPA (enzyme linked inorganic phosphate assay) and a radio-labeled assay. In the ELIPA, pyrophosphate released during the condensation of DHPPP and $p$ ABA is broken down into inorganic phosphate by a pyrophosphatase. The inorganic phosphate then reacts with 2-amino-6-mercapto-7-methylpurine riboside (MESG) to produce mercaptomethylpurine and ribose-1-phophate. Since the absorption ranges of MESG (330 nm) and mercato-methylpurine $(360 \mathrm{~nm})$ are different, the amount of pyrophosphate released can be measured by observing an increase in absorption at $360 \mathrm{~nm}$ over time. Therefore, the 
results of the ELIPA experiment correlate to the amount of phosphate released during catalysis. Two reactions were tested by this method: the reaction DHPPP $+p \mathrm{ABA}$ and the reaction of DHPPP + SMX (sulfamethoxazole) in the absence of $p$ ABA. As shown in Figure 4.1, both reactions show a comparable release of phosphate indicating that SMX is able to undergo enzymatic catalysis. While these results reveal a unique characteristic of the sulfonamide class of drugs, they limit the use of the ELIPA experiment for studies in antibiotic resistance. To overcome this issue, we developed a radio-labeled assay using ${ }^{14} \mathrm{C} p \mathrm{ABA}$ as one of the substrates and monitoring the appearance of ${ }^{14} \mathrm{C}$-labeled product by thin-layer chromatography (Nopponpunth et al., 1999; Kasekarn et al., 2004).

The radiometric assay was used to measure the kinetic parameter $\mathrm{K}_{\mathrm{m}}$ and $\mathrm{K}_{\mathrm{cat}}$ of mutated DHPS enzymes. Several types of sulfonamide resistance determinants have been identified (Sköld, 2000; 2001). Five point mutations were identified and chosen for this study (P69S, T67A, R68A, G188C, and F33L) based on the relative position to either Loop-1 or Loop-2. Each mutated strain was mutated from the WT strain of B. anthracis using site-directed mutagenesis as described in Material and Methods. Mutations P69S, T67A, R68A, and G188C all show an increase in $\mathrm{K}_{\mathrm{m}}$ and $\mathrm{K}_{\text {cat }}$ values as compared to the WT enzyme indicating that the enzyme must lower efficacy to accommodate sulfonamide resistance (Table 4.1). The mutation F33L showed no activity in vitro as compare to the WT enzyme (Table 4.1; Figure 4.2). However, an extensive literature search yields reports of clinical isolates with this specific mutation (Dallas et al., 1992; Fermer \& Swedberg, 1997). Furthermore, these isolates were obtained from sulfonamide resistance, pathogenic strains (Dallas et al., 1992; Fermer \& Swedberg, 1997; Lane et al., 1997).

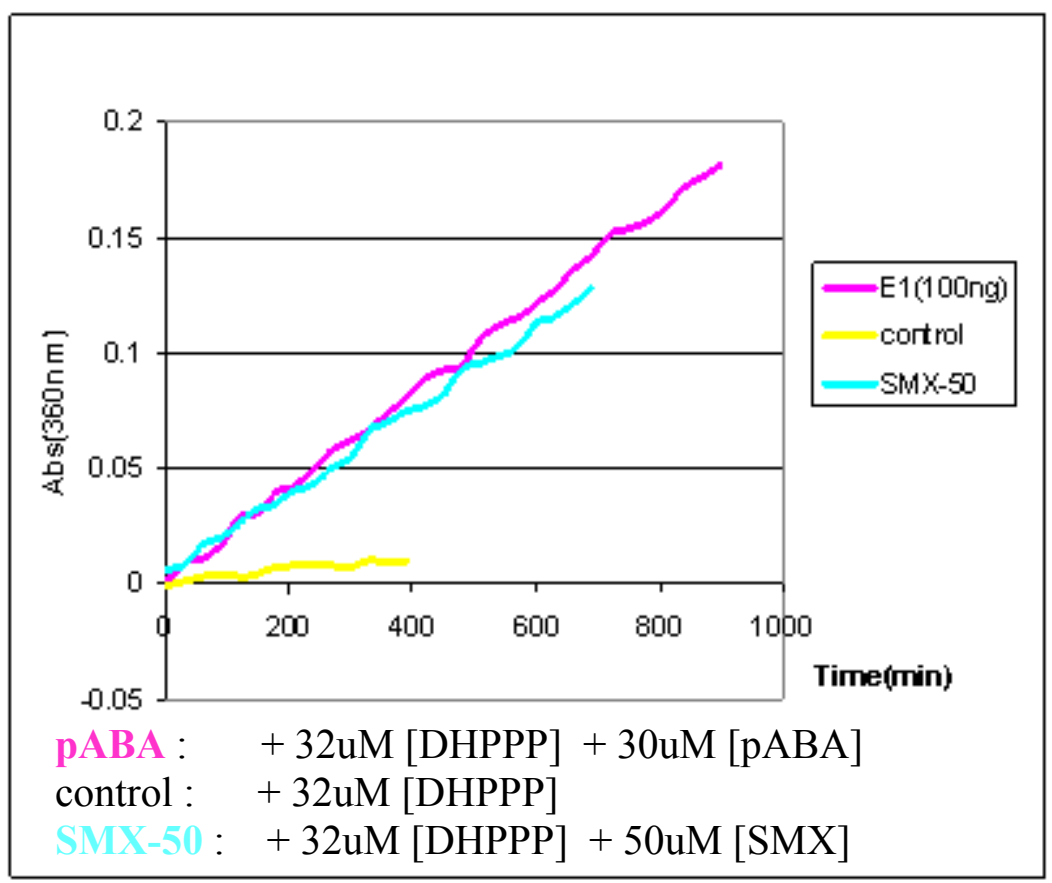

Figure 4.1: Results from the ELIPA Experiment. 
Table 4.1: Kinetics Studies of Mutant Alleles.

\begin{tabular}{|c|c|c|c|c|c|c|}
\hline \multirow[b]{2}{*}{ Construct } & \multirow[b]{2}{*}{$\mathrm{K}_{\mathrm{m}}(\mu \mathrm{M})$} & \multirow[b]{2}{*}{$\mathrm{K}_{\mathrm{cat}}\left(\mathrm{min}^{-1}\right)$} & \multicolumn{2}{|c|}{ SMX } & \multicolumn{2}{|c|}{ SNA } \\
\hline & & & $\mathrm{IC}_{50}(\mu \mathrm{M})$ & $\mathrm{K}_{\mathrm{i}}(\mu \mathrm{M})$ & $\left.\mathrm{IC}_{50}(\mu \mathrm{M})\right)$ & $\mathrm{K}_{\mathrm{i}}(\mu \mathrm{M})$ \\
\hline WT & $0.3370( \pm 0.1263)$ & $21.82( \pm 2.17)$ & $2.258( \pm 0.232)$ & $0.143( \pm 0.014)$ & $18.52( \pm 1.72)$ & $1.169( \pm 0.108)$ \\
\hline P69S & $5.751( \pm 2.454)$ & $7.000( \pm 1.43)$ & $5.196( \pm 0.434)$ & $2.779( \pm 0.232)$ & $39.88( \pm 3.61)$ & $21.33( \pm 1.93)$ \\
\hline T67A & $0.5823( \pm 0.200)$ & $11.16( \pm 1.02)$ & $10.26( \pm 0.95)$ & $1.070( \pm 0.099)$ & $315.5( \pm 72.0)$ & $32.91( \pm 7.50)$ \\
\hline R68A & $1.523( \pm 0.719)$ & $13.43( \pm 2.31)$ & $0.881( \pm 0.064)$ & $0.206( \pm 0.015)$ & $13.42( \pm 1.406)$ & $3.133( \pm 0.328)$ \\
\hline G188C & $0.699( \pm 0.250)$ & $7.29( \pm 0.83)$ & $7.1754( \pm 1.653)$ & $0.880( \pm 0.203)$ & $31.24( \pm 5.96)$ & $3.831( \pm 0.731)$ \\
\hline F33L & $>50$ & $\mathrm{~N} / \mathrm{A}$ & $\mathrm{N} / \mathrm{A}$ & $\mathrm{N} / \mathrm{A}$ & N/A & $\mathrm{N} / \mathrm{A}$ \\
\hline
\end{tabular}




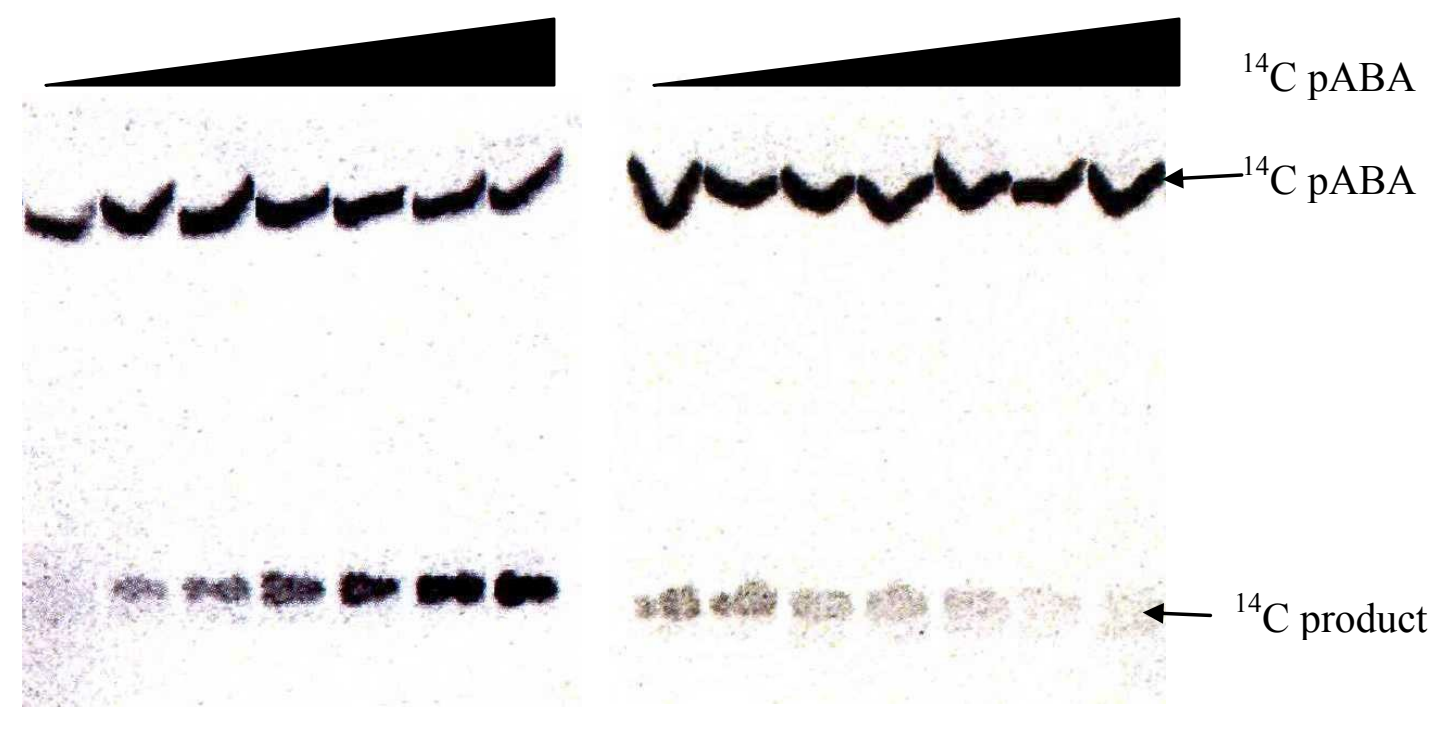

Figure 4.2: Results from the Radiometric Assay.

In order to understand our results, we verified that the mutation was successful. To do so, we isolated the plasmid of interest and sequenced the gene by the dideoxy chain-termination method for sequencing DNA. These results were then aligned with the wild type sequence and analyzed for the appearance of mutations. As seen in Figure 4.3, the alignment revealed that the triad TTT encoding for Phenylalanine 33 was indeed mutated to a CTG encoding for a Leucine. Therefore, the mutation was correct and the results observed in the radiometric assay correlate to a F33L mutation.

In addition to $\mathrm{K}_{\mathrm{m}}$ and $\mathrm{K}_{\text {cat }}$ studies, $\mathrm{IC}_{50}$ values in the presence of sulfamethoxazole (SMX) were determined for each mutant enzyme as well as $\mathrm{IC}_{50}$ values in the presence sulfanilamide (SNA). Mutations P69S, T67A, R68A, and G188C all showed an increase in $\mathrm{IC}_{50}$ values as compared to WT in the presence of both SMX and SNA (Table 4.1). However, the ability was amplified against SNA as compared to SMX indicating that the terminal ring of SMX may be hindering the enzyme's ability to develop drug resistance while maintaining a viable enzyme. This data showed that the T67A mutation has the most pronounced effect upon developing drug resistance while having the least effect on the catalytic efficacy of the enzyme. In contrast, R68A and P69S mutations seem to offer little sulfonamide resistance in light of a five fold or better loss in the catalytic efficacy. These results yield a great deal of insight into the catalytic mechanism of the enzyme as well as key residues necessary for catalysis, namely P69, R68, and F33 all of which are highly conserved residues in the DHPS sequence.

In addition to the kinetic studies, we have performed preliminary structural studies of the mutant alleles. We have successfully crystallized P69S, F33L, and T67A mutants (Figure 4.4), but we have not been able to visualize Loops-1 or -2 in these crystal 


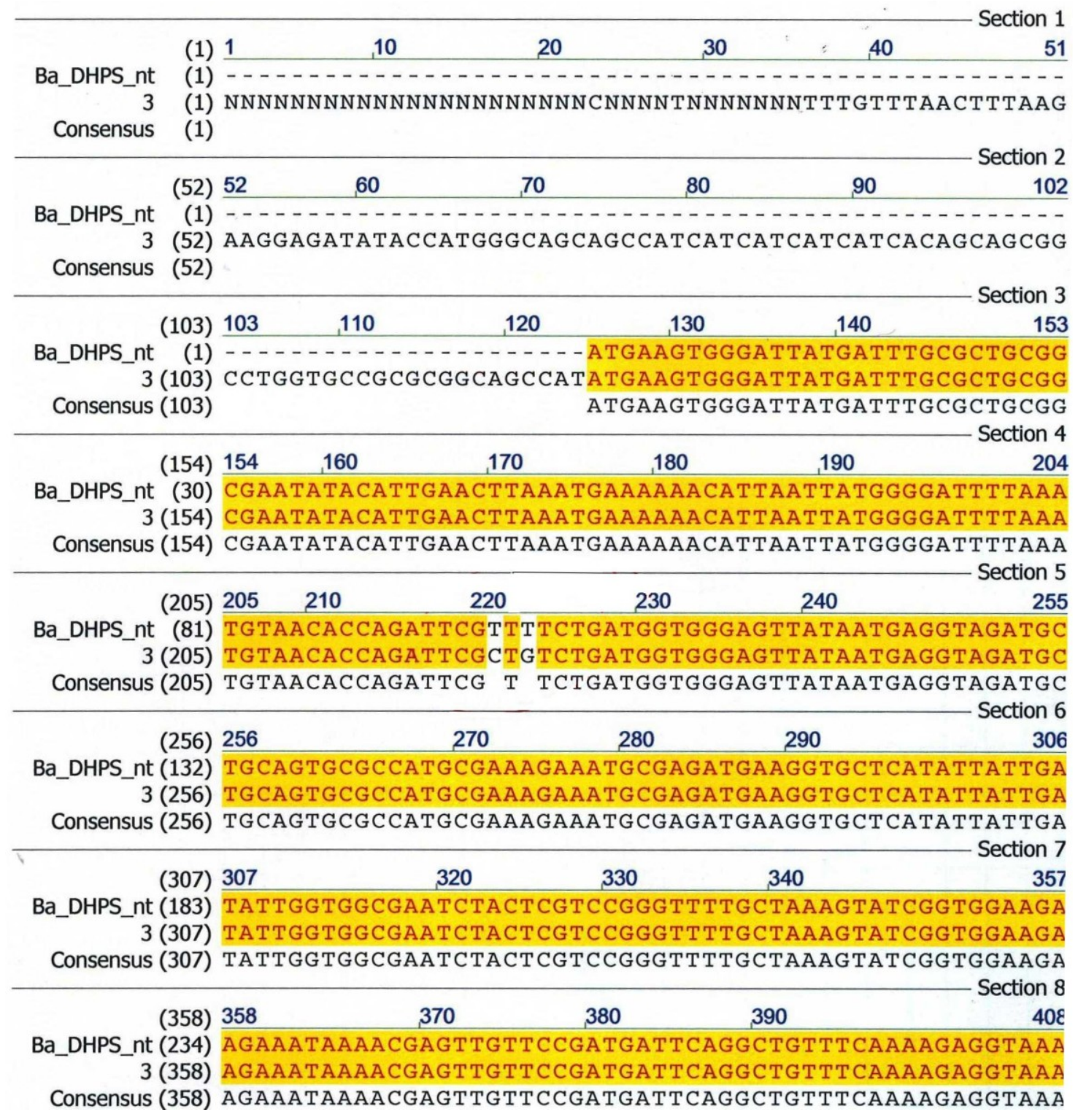

Figure 4.3: F33L Sequence Verification. 


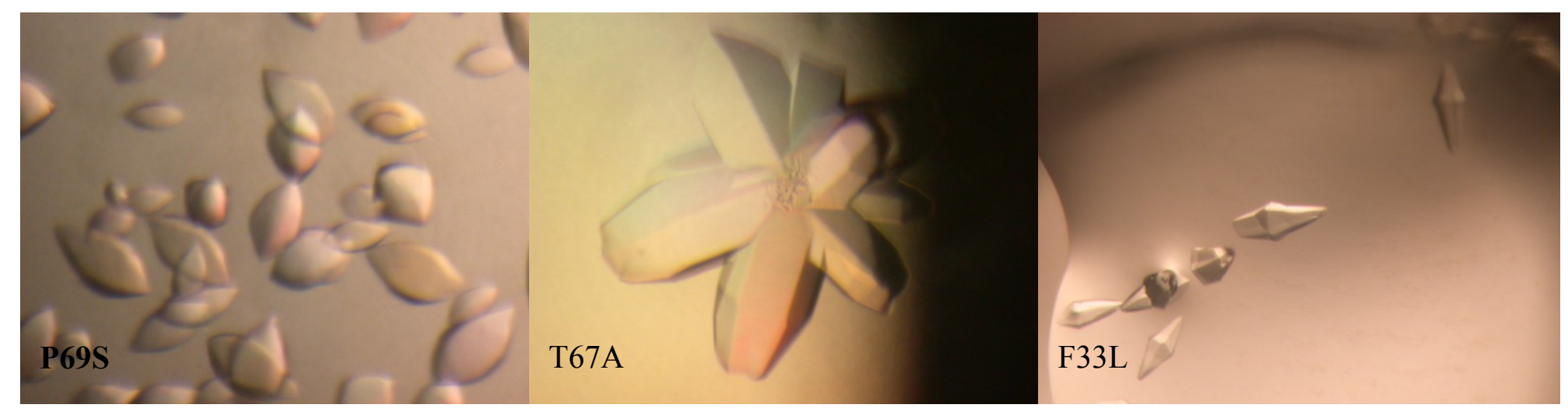

Figure 4.4: Crystallization of the P69S, T67A, and F33L Mutant Alleles. 
structures. Attempts have been made to co-crystallize these mutant enzymes with pteroic acid, but with no success to date. 


\section{Chapter 5: Discussion}

\section{The Conformation and Roles of Loops-1 and -2}

In all known crystal structure of DHPS, including the double mutant construct reported in this thesis, Loops-1 and -2 adopt highly variable conformations or are missing all together. This observation suggests that these loops are highly mobile and work in an ordered manner to aid in substrate binding and stabilization. As regards to Loop-1, we can offer no new structural insights into the why it is so conserved. Based on the $M$. tuberculosis structure, Loop-1 can fold in such a way as to allow key residues such as Asp35 to interact with the $p$ ABA binding sight. Our kinetics data reiterates the importance of Loop- 1 for $p$ ABA binding by revealing that Phe33 is essential to the catalytic efficacy of the enzyme.

The conformation of Loop-2 that we present in the DHPS double mutant construct is unique amongst the known DHPS structures. By modeling in the pteroic acid product analogue, our structure reveals that the side chain of conserved Arg68 bends towards the carboxylic acid component of $p \mathrm{ABA}$ to stabilize it in the binding site. Babaoglu et al. reports that $\operatorname{Arg} 68$ folds into the pterin binding sight to give added stability to the unliganded enzyme. This report in conjunction with our data suggests that Arg68 may function to ensure $p \mathrm{ABA}$ can only bind if the pterin substrate is already bound into the active sight. The suggested binding of pABA between ordered Loops- 1 and -2 would essentially block the pterin pocket, thereby preventing pterin from binding into the active site. This is further confirmed by previous reports that enzyme catalysis proceeds via an ordered mechanism in which bound pterin is required for $p \mathrm{ABA}$ binding (Vinnicombe \& Derrick, 1999; Levy et al., 2008). Our structure shows for the first time a functionally significant conformation of Loop-2 and the role of Arg68 in enzyme catalysis.

\section{Implications of Sulfonamide Resistance}

Antibiotic resistance is the ability of a microorganism to overcome the effects of a particular drug due to genome modifications. This process occurs via natural selection in which random point mutations lead to natural selection of a drug resistant gene. Such genes can then be inserted in to the pathogen genome via horizontal gene transfer (Ochiai et al., 1959). Introduction of antibiotic action against a pathogen creates an environmental stress in the system. Bacteria which have the mutation allowing them to survive will reproduce ensuring that the advantageous gene mutation is passed on to their offspring (Figure 5.1). The result is a fully resistant colony.

Microorganisms are able to exhibit antibiotic resistance in a variety of ways. The four most common modes of resistance development are as follows: drug inactivation or modification, alteration of target site, alteration of metabolic pathway, and reduced drug accumulation. 


\section{Initial Population}

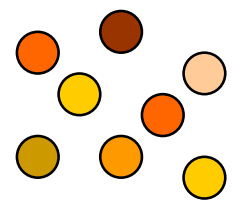

\section{Exposure to Environmental Stress}

(Antibiotic)

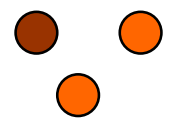

Final Population

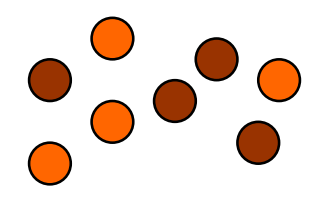

Resistance Level

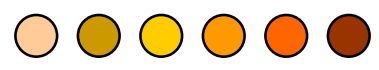

Figure 5.1: Schematic Representation of the Formation of Antibiotic Resistant Colonies. 
This study clearly shows that specific point mutations in the target site of sulfonamide binding leads to a decrease in binding affinity of sulfonamides. Specifically, mutations which disrupt the structural stability of Loops-1 and -2 have significant effects on drug binding which ultimately lead to antibiotic resistant bacterial strains. This evidence suggests that these flexible loop regions of the DHPS enzyme can readily accommodate point mutations while maintaining a functionally viable enzyme. Therefore, it is important to avoid such regions during the development of novel therapeutics which target the DHPS enzyme.

\section{The Catalytic Mechanism of DHPS}

By docking in the pteroic acid structure into our new DHPS structure with a functionally significant conformation of Loop-2, it is possible to generate a model of the DHPS catalytic mechanism (Figure 3.8). This model shows that SMX binds to the DHPS active site in a similar fashion as $p \mathrm{ABA}$ and this interaction is facilitated by $\operatorname{Arg} 68$ as well as additional components of Loop-2. Furthermore, the position of SMX in the active site reiterates the proposed SN2 mechanism previously reported by Babaouglu and colleagues.

Based on previous data in conjunction with the data presented in this thesis, we propose that the mechanism proceeds by four steps via an ordered mechanism. In the initial state, Arg68 is bound into the pterin pocket as seen by Babaouglu and colleagues. During the first step, DHPP binds into the active site (Vinnicombe \& Derrick, 1999; Levy et al., 2008) displacing Arg68 from the pterin pocket. This causes a conformational shift of Loop-2 which can easily be envisaged by comparing our new Loop-2 conformation with those of previously reported conformations in the $E$. coli and $S$. aureus structures. Facilitated by the pterin moiety, the $\beta$-phosphate enters the anion binding pocket and the divalent cation binding site involving Asn27 from Loop-1 becomes occupied by the $\mathrm{Mg} 2+$ ion that coordinates the pyrophosphate for an SN2 reaction. Indeed, the divalent cation may aid in drawing Loop-1 into the active site and thereby bringing Phe33 with it. The second step creates the $p \mathrm{ABA}$ binding site. Formation of the $p \mathrm{ABA}$ binding site is facilitated by the binding of the DHPP and the binding of Lys220 to the pterin moiety. Lys 220 stretches to form a platform by which $p$ ABA can dock into the active site. As Lys220 swings out it exposes the N-terminal end of helix $\alpha$-Loop- 7 for additional hydrogen binding interactions. The $p \mathrm{ABA}$ binding site is completed by movement in Loops-1 and -2, and Arg68 in its new conformation seen in the Pt-SMX structure now interacts with $p \mathrm{ABA}$ via charge interactions and $\pi$-stacking interactions. The third step involves nucleophilic SN2 attack by the nitrogen group of $p \mathrm{ABA}$ on the methyl carbon on the C6 position of DHPP. The divalent ion coordinates the pyrophosphate and functions to make it a better leaving group facilitating an SN2 attack. During the final step, Loop-2 reinserts into the pterin pocket and product is released for further modification down the metabolic pathway. The enzyme is then ready to repeat the enzymatic mechanism with new substrate. Table 5.1 depicts all the residues believed to be involved in the catalytic mechanisms as well as synonymous residues in different organisms. The residues colored red represent those with sequence variance as compared 
Table 5.1: Sidechains Involved in Substrate Binding.

\begin{tabular}{|c|c|c|c|c|c|c|}
\hline B. anthracis & Interaction Type & E. coli & S. aureus & M. tuberculosis & S. pneumoniae & P. aeruginosa \\
\hline Thr67 & & Thr62 & Thr51 & Ser53 & Thr57 & Ser49 \\
\hline Arg68 & H-donor/ Pi Electronics & Arg63 & Arg52 & Arg54 & $\operatorname{Arg} 58$ & His 50 \\
\hline Asp101 & & Asp96 & Asp84 & Asp86 & Asp91 & Asp82 \\
\hline Asn120 & H-acceptor & Asn 115 & Asn 103 & Asn 105 & Asn 110 & Asn101 \\
\hline Ile122 & vDW & Ile117 & Gln105 & Val107 & Ile 112 & Ile103 \\
\hline Ile143 & vDW & Cys 137 & Val126 & Val128 & Val133 & Val123 \\
\hline Met145 & Pi Electronic & Met139 & Met128 & Met130 & Met135 & Met125 \\
\hline Asp184 & H-acceptor & Asp185 & Asp167 & Asp177 & Asp201 & Asp173 \\
\hline Phe189 & Pi Electronic & Phe190 & Phe172 & Phe182 & Phe206 & Phe178 \\
\hline Leu214 & vDW & Leu215 & Leu197 & Leu207 & Phe231 & Leu206 \\
\hline Gly216 & & Gly217 & Ala199 & Gly209 & Gly 233 & Ser208 \\
\hline Lys 220 & H-donor & Lys 221 & Lys 203 & Lys 213 & Lys 237 & Lys 212 \\
\hline Arg254 & Pi Electronic & Arg255 & Arg239 & Arg253 & Arg282 & Arg246 \\
\hline
\end{tabular}

Note: Residues in red are those that diverge from the B. anthracis sequence 
to the $B$. anthracis sequence. In addition, the table shows the types of interactions each residue participates in upon substrate binding. 


\section{Chapter 6: Materials and Methods}

\section{Cloning of the folP Gene}

The anthrax folP gene encoding DHPS was obtained by PCR amplification using genomic DNA obtained from the Sterne 34F2 strain of Bacillus anthracis as described in Babaouglu et al., 2004. The following primers, with inserted restriction sites in italics, were designed using the sequence from the A2102 strain deposited in the NCBI database (gi:21397375): 5' NdeI: CATATGAAGTGGGATTATGATTTGCGC and 3' BamHI: GGATCCTTACTTTACCCCTTACCAATCATCGC.

Three reactions were set up using the High Fidelity supermix kit (Invetrogen, Carisbad, CA) and the protocol suggested in the product literature. The PCR reaction consisted of an additional heating step for $2 \mathrm{~min}$. at $94^{\circ} \mathrm{C}$ followed by 25 cycles of $30 \mathrm{sec}$. at $94^{\circ} \mathrm{C}$, annealing at $53^{\circ} \mathrm{C}$ for $30 \mathrm{sec}$., and extension at $72^{\circ} \mathrm{C}$ for $2 \mathrm{~min}$. The PCR products were analyzed on a $1 \%$ agarose gel stained with ethidium bromide to identify the amplification species of the appropriate size ( $846 \mathrm{bp})$. The PCR products were purified, subcloned using the TOPO TA cloning kit (Invitrogen, Carlsbad, CA) into the pCR 2.1 TOPO vector, and finally sequenced to ensure that the restriction during the PCR reactions. Primer synthesis and DNA sequencing were performed by the Hartwell Center at St. Jude Children's Research Hospital.

\section{Protein Expression and Purification}

The folP gene encoding DHPS was obtained and subcloned from the TOPO TA vector into the $\mathrm{pET}$-28a expression plasmid (Novagen, Madison, WI) that incorporates a 6xHis Tag at the $\mathrm{N}$ terminus of the expressed protein (Babaoglu et al., 2000). Two

surface residues were mutated using Quickchange ${ }^{\circledR}$ (Stratagene, La Jolla, CA) sitedirected mutagenesis. The plasmid was then transformed into competent BL21 (DE3) E. coli cells (Stratagene) for expression. A $100 \mathrm{~mL}$ overnight culture was grown form a single colony of cells in LB containing $50 \mu \mathrm{g} / \mathrm{ml}$ kanamycin, and this culture was used to inoculate $6 \mathrm{~L}$. of the same medium. This was grown at $37^{\circ} \mathrm{C}$ until the $\mathrm{OD}_{600}$ reached $\sim 0.6$, at which point isopropyl- $\beta$-D-thiogalactopyranoside (IPTG) was added to a final concentration of $1 \mathrm{mM}$. Cell were then allowed to grow overnight at $16^{\circ} \mathrm{C}$ in the presence of IPTG and then harvested by centrifugation. The cell pellet was resuspended in $50 \mathrm{ml}$ lysis buffer (20 mM NaPO $4, \mathrm{pH} 8.0,0.5 \mathrm{mM} \mathrm{NaCl}$, and $1 \mathrm{mM}$ Imidazole). The cells were lysed by a microfluidizer, and the cell debris was cleared by centrifugation at $30,000 \mathrm{xg}$ for 1 hour.

Purification of DHPS required two steps of Fast Performance Liquid Chromoatography (FPLC), Ni2+ chelation affinity chromatography and gel filtration. The cell lysate was first applied to a $5 \mathrm{ml}$ HiTrap Chelating HP column (Amersham Biosciences, Piscataway, NJ) and washed with ten column volumes of lysis buffer. The protein eluted during a gradient of $0-0.3 \mathrm{M}$ imidazole, and the appropriate fractions were 
identified by SDS-PAGE. The pooled DHPS was concentrated to $3 \mathrm{ml}$, applied to a HiLoad 16/60 Superdex 75 column (Amersham Biosciences), and eluted with running buffer (20 $\mathrm{mM} \mathrm{NaPO}_{4}, \mathrm{pH} 8.0,0.2 \mathrm{M} \mathrm{NaCl}, 1 \mathrm{mM}$ dithiothreitol (DTT), and $1 \mathrm{mM}$ $\mathrm{MgCl}_{2}$ ) at a flow rate of $0.5 \mathrm{ml} / \mathrm{min}$. The final purified protein was estimated to be $>95 \%$ pure by SDS-PAGE analysis.

\section{Crystallization and Data Collection}

The purified enzyme was concentrated with an Amicon stirred cell to a final concentration of $10 \mathrm{mg} / \mathrm{ml}$. Using the hanging drop vapor diffusion method together with Hampton Crystal Screens kits (Aliso Viejo, CA), a number of initial crystallization conditions were identified by mixing equal volumes $(2 \mu \mathrm{l})$ of protein to well solution. These conditions were subsequently optimized, and large well-diffracting crystals could eventually be grown from 1.8 M Sodium Malonate. Large rectangular box shaped crystals could be obtained within $72 \mathrm{hrs}$ at $18^{\circ} \mathrm{C}$. The crystals are in space group $\mathrm{P} 22_{1} 2_{1} 2_{1}$ with cell dimensions $\mathrm{a}=100.357 \AA, \mathrm{b}=107.999 \AA, \mathrm{c}=243.443 \AA$. Consideration of the Mathews coefficient suggested that eight molecules of DHPS should be present in the asymmetric unit, and this was subsequently confirmed. For the structural studies of pterin-sulfamethoxazole (Pt-SMX), the compounds were directly added to pregrown crystals in the form of saturated solution in the crystallization buffer, and allowed to soak for $72 \mathrm{hrs}$.

All x-ray diffraction data were collected under cryogenic conditions. Crystals were briefly immersed in a cryoprotectant mixture of $25 \%$ glycerol and directly flash frozen in liquid nitrogen. All data were collected at the Southeast Regional Collaborative Access Team (SER-CAT) synchrotron beamline 22-ID and 22-BM at the Advanced Photon Source (Argonne National Laboratory). All crystals were frozen and prescreened in-house before synchrotron data collection. The DHPS-PtSMX synchrotron data were collected on a MAR300 CCD detector and processed and merged using HKL2000 (Otwinowski \& Minor, 1997). All relevant data collection statistics are shown in Table 3.2 .

\section{Structure Determination}

The structure of the double mutant DHPS from B. anthracis was determined by molecular replacement using the CCP4 program Phaser (McCoy et al., 2007). Monomer A of the wild-type $B$. anthracis structure (PDB: 1TWS) was used as an initial search model. A solution was obtained using high resolution synchrotron data and refined following several iterative rounds of rebuilding in COOT (Emsley \& Cowtan, 2004), and simulated annealing and temperature factor refinement using CNS (Brüger et al., 1998). All the waters were picked automatically using COOT and subsequently confirmed by visual inspection. Finally, to verify ambiguous regions of the electron density and to check the model, a number of simulated annealing omit maps were calculated during the later stages of the refinement process. The quality of the structure was verified using 
PROCHECK (Laskowski et al., 1993), and the final refinement statistics are shown in Table 3.1.

\section{Radiometric Assay}

To observe enzyme kinetics, we developed a radio-labeled assay using ${ }^{14} \mathrm{C} p \mathrm{ABA}$ as one of the substrates and monitoring the appearance of ${ }^{14} \mathrm{C}$-labeled product by thinlayer chromatography (Nopponpunth et al., 1999; Kasekarn et al., 2004). For the first reaction (synthesis of DHPPP), the substrate (10 $\mu \mathrm{M}$ of HMDP) was incubated in presence of ATP $(250 \mu \mathrm{M}), \mathrm{MgCl}_{2}(10 \mathrm{mM})$, and HPPK enzyme $(10 \mu \mathrm{g})$ at $37^{\circ} \mathrm{C}$ for 30 minutes. The reaction solution was mixed with GST resin, and the supernatant was collected for the second step of the reaction. DHPS activity was measured in a $50 \mu \mathrm{L}$ reaction containing $50 \mathrm{mM}$ HEPES ( $\mathrm{pH} 7.6$ ), $10 \mathrm{mM} \mathrm{Mg} \mathrm{Cl2,1 \%} \mathrm{PEG} \mathrm{400,} \mathrm{and} \mathrm{2 \%}$ DMSO. For $\mathrm{K}_{\text {cat }}$ and $\mathrm{K}_{\mathrm{m}}$ determination, varying concentrations of DHPS, $0-3 \mu \mathrm{M}{ }^{14} \mathrm{C}$ $p \mathrm{ABA}$, and $10 \mu \mathrm{M}$ DHPPP from the first reaction were added to the reaction mixture. For $\mathrm{K}_{\mathrm{i}}$ and $\mathrm{IC}_{50}$ determination, varying amounts of DHPS, $5 \mu \mathrm{M}{ }^{14} \mathrm{C} p \mathrm{ABA}, 10 \mu \mathrm{M}$ DHPPP and $0-50 \mu \mathrm{M}$ of SMX were added to the reaction mixture. The reaction solution was incubated at $37^{\circ} \mathrm{C}$ for 30 minutes. The labeled product of the enzymatic reaction was separated from the labeled $p \mathrm{ABA}$ by thin layer chromatography. $25 \mu \mathrm{L}$ aliquots of the reaction mixture were spotted onto PEI-cellulose coated plates and developed with ascending chromatography in $100 \mathrm{mM}$ phosphate buffer, $\mathrm{pH}$ 7.4. With this method, we are able to perform Michealis-Menten kinetics for wild type and mutant enzyme activity as well as determine the $\mathrm{IC}_{50}$ values of candidate inhibitors. Since sulfonamides are competitive inhibitors of DHPS, $\mathrm{K}_{\mathrm{i}}$ values were determined using the Cheng-Pursoff equation and represent the amount of inhibitor that will bind to half the enzyme active sites at equilibrium in the absence of substrate (Cheng \& Prusoff, 1973). Enzyme kinetics data were determined by nonlinear regression using the Graphpad Prism4 software. 


\section{Chapter 7: Future Directions and Conclusions}

\section{Developing a Robust Enzyme Assay}

While our current assays are effective for interpreting changes in $K_{M}$ which occur as a result of sulfonamide-resistance mutations, the formation of sulfonamide products makes $\mathrm{IC}_{50}$ and $\mathrm{K}_{\mathrm{i}}$ values difficult to interpret as DHPPP depletion may alter inhibition in a way that does not correlate physiologically. Therefore, a more robust method for studying sulfonamide inhibition is essential for understanding the mode of enzyme resistance. To overcome these issues, we propose the use of stopped-flow transient kinetics.

Stopped-flow fluorescence spectroscopy is a useful tool for determining the dissociation constant $\left(\mathrm{K}_{\mathrm{d}}\right)$ for individual substrates. This method is particularly useful in systems, such as the DHPS system, with intrinsically fluorescent substrates. Changes in the fluorescence of the pterin ring as well as $p$ ABA have been used to measure binding of substrates in both wild-type and sulfonamide resistant enzymes (Levy et al., 2008). We will use similar methods as described in Levi et al. to fully characterize the effects of sulfonamide resistance mutations on substrate and inhibitor binding. In addition, fluorescence probes can be used to understand conformational shifts which occur in loop regions of DHPS to facilitate substrate binding. Intrinsic Tryptophan fluorescence is responsible for the majority of fluorescence observed in proteins. A sequence analysis of the wild-type $B$. anthracis DHPS revealed only two Tryptophan residues which are far removed from the enzyme active site. Since neither residue is conserved, it stands to reason that they can easily be mutated out with no consequence to enzyme integrity. This frees the enzyme of unwanted fluorescence and allows the use of Tryptophan as a fluorescence probe. We will use site-directed mutagenesis to place Tryptophan residues in strategic regions of the enzyme to better understand the role of Loops- 1 and -2 and their involvement in substrate binding.

While it has been shown that pyrophosphate is essential for $p$ ABA binding (Levy et al., 2008), it has yet to be shown whether or not pyrophosphate is required for catalytic function. This is critical for understanding the catalytic mechanism as the presence or absence of pyrophosphate is directly linked to the nature of the transition-state intermediate. In the event of an $\mathrm{SN} 1$ reaction, pyrophosphate is released to form a carbocation intermediate prior to attack by $p$ ABA. However, pyrophosphate is required for the formation of the SN2 transition-state intermediate as proposed by Baca et al. and release of pyrophosphate in the absence of a nucleophilic attack can only occur as a result of an SN1 mechanism. Therefore, we will use inactive substrates which mimic $p$ ABA, such as benzoic acid, and monitor the release of pyrophosphate using our enzyme linked inorganic phosphate assay (ELIPA). This approach will provide direct evidence as to the nature of the enzyme transition-state as well as a model for understanding the catalytic mechanism. 
In the ELIPA, pyrophosphate released during the condensation of DHPPP and $p \mathrm{ABA}$ is broken down into inorganic phosphate by a pyrophosphatase. The inorganic phosphate then reacts with 2-amino-6-mercapto-7-methylpurine riboside (MESG) to produce mercapto-methylpurine and ribose-1-phophate. Since the absorption ranges of MESG $(330 \mathrm{~nm})$ and mercato-methylpurine $(360 \mathrm{~nm})$ are different, the amount of pyrophosphate released can be measured by observing an increase in absorption at 360 nm over time.

In addition to our in vitro assays, we will use a DHPS knockout strain (JW3-1441; Baba et al., 2006) of E. coli to determine the minimal inhibitory concentration (MIC) of sulfonamides as a means of analyzing the effects of sulfonamide resistance mutations on bacterial growth. Using the $\mathrm{pBAD} /$ His (Invitrogen) plasmid system, we will insert our DHPS gene of choice into the knockout cells. The $\mathrm{pBAD} /$ His system is advantageous because it provides a low copy system (pBR322 origin) with a leaky promotor (araC ORF) which will allow for approximate basal level expression of DHPS and DHPS mutant enzymes with no additional stimuli required. Two positive controls will be required for this experiment. First, transformation of the DHPS knockout strain with wild-type DHPS will provide a baseline for comparing MICs. Second, a DHPS positive strain of $E$. coli will be used to determine wild-type MICs which may be altered in our plasmid system due to over expression of the plasmid inserts. A negative control will also be provided in which the DHPS knockout strain will be transformed with an empty plasmid. It should be noted that DHPS knockout strains of $E$. coli will require folate supplements. However, once the DHPS plasmid is transformed into the system supplements will be removed from the media and should not effect MIC determination. This is a widely used technique for understanding drug resistance (Rock et al., 2003; Zhang et al., 2004)) and will provide a more accurate method for evaluating the effects of drug resistance.

\section{Probing the Enzyme Mechanism}

With the introduction of kits like Quickchange ${ }^{\circledR}$ (Stratagene), site-directed mutagenesis has become a relatively straightforward method for designing point mutations. This technique will be of great importance for the advancement of this project as it will allow us to probe the enzyme active site for catalytically significant residues. Several residues have already been identified and will be mutated in ways as to both diminish and enhance catalytic activity according to predicted function. For instance, Asparagine 27 ( $\mathrm{B}$. anthracis) is predicted to coordinate the divalent cation which is predicted to protonate the pyrophosphate leaving group. Therefore, mutating Asparagine 27 to an Alanine should diminish catalytic activity of the enzyme where as mutation to a Histidine should restore enzyme function.

The current understanding of the DHPS mechanism assumes an SN2 transition state. However, conflicting data in the literature as to the direction of the nucleophilic attack requires further investigation into the nature of the enzyme transition-state. To distinguish between a SN2 and a SN1 mechanism, we have analyzed the enzyme active 
site to identify conserved residues which aid in transition-state stabilization. In order for the reaction to proceed via $\mathrm{SN} 1$, the enzyme must have a way of stabilizing a carbocation intermediate. Aspartate 35 (B. anthracis) is a highly conserved residue on Loop-1 and is predicted to stabilize the formation of a carbocation by electrostatic interactions in the event of a SN1 reaction. In contrast, it is a less crucial candidate for an SN2 transitionstate because there is no longer a positive charge on the pterin moiety to be neutralized. Therefore, mutating Aspartate 35 to an Alanine or a lysine will provide critical information pertaining to the nature of the enzyme transition state.

In addition, our preliminary data shows that Loop- 2 folds into the active site upon substrate binding. Furthermore, disruption of the rigidity of Loop-2 by mutating of Proline 69 (B. anthracis) to a Serine diminishes catalytic activity by 3-fold. Evidence suggests that Loop-2 is necessary to direct Arginine 68 (B. anthracis) into the active site as mutation of Arginine 68 to an Alanine yields a 2-fold reduction in the rate of catalysis. To further understand the role of Loop-2, we will perform a series of mutations using an Alanine probe along Loop-2 as well as removal of Loop-2 to observe the effects on catalysis using our radiometric assay. In conjunction with our radiometric assay, we will determine effects of Loop-2 mutagenesis on pyrophosphate as well as $p$ ABA binding utilizing fluorescence techniques described by Levy et al.

\section{Fragment-Based Virtual Screening}

Structural data suggests that the $p \mathrm{ABA}$ and pterin binding sites are separate, distinct regions in the DHPS molecule (Achari et al., 1997) allowing for the development of inhibitors capable of inhibiting sulfonamide-resistant forms of DHPS. Since the pterin binding pocket is highly conserved, it seems logical that it would be more difficult for the enzyme to accommodate mutations against pterin-based compounds. In addition, mutations that could potentially cause resistance to pterin-based inhibitors are likely to disrupt structural integrity since the pterin binding site is buried within the protein. In contrast, the $p \mathrm{ABA}$ binding site involves flexible loop regions near the surface of the protein, and the rapid emergence of multiple sulfonamide resistance mutations clearly show that they are easily tolerated within the overall structure of the protein. By targeting the pterin binding site, we hypothesize that we will be able to overcome the problems of sulfonamide resistance which have arisen by mutations in the $p$ ABA binding site.

While we have successfully identified several target compounds, the majority of these compounds have been pterin-like compounds which are often insoluble. To overcome this issue, we will use fragment-based virtual screening to identify scaffold compounds for lead optimization to produce highly efficient, drug-like inhibitors. The general approach to this aim will be structure-based drug design techniques in which virtual screening is used in synergism with structural and biochemical data.

Fragment-based drug discovery is a highly integrated process which begins with the screening of fragment libraries and utilizes structural and biochemical techniques to produce drug-like compounds. We will adopt a modified rule of three in which library 
searches are limited to compounds with a molecular weight less than 350 and no more that 5 rotatable bonds. Pharmacophore constraints will be applied based on substrate binding, but these will be limited to partial match derivatives to loosen the macro constraints and allow for ligand diversity. The hit compounds will be docked and scored using the methods described in preliminary data. Of the highest scoring compounds, the top $1 \%$ will be moved to biochemical studies.

We will use our radio-labeled assay as our primary method for studying inhibition. In contrast to the sulfonamides, these compounds will not be able to undergo enzyme catalysis, and we predict that this method will be an acceptable assay for inhibition kinetics. However, the ELIPA assay is available should an alternative be required. Compounds with greater than $30 \%$ inhibition of the wild-type DHPS will be moved into structural studies.

Visualization of these compounds is an essential step in our approach as it will allow us to see how the fragment compounds bind into the pterin pocket. This is advantageous for two reasons. First, it provides a model by which we can optimize these fragments into lead compounds with drug-like properties. Second, it provides a validation scheme for our docking and scoring functions. As a result of these studies, we will identify several lead compounds as candidates for novel therapeutics to be used against a wide variety of infectious agents.

Although we predict that the pterin pocket is less likely to accommodate resistance mutations, we cannot deny that this is a possibility. Therefore, we will grow $E$. coli cultures in agar containing varying amounts of drug concentration and observe for growth. The DHPS gene (folP) from the genomic DNA of cultures found to grow in the presence of drug will be PCR (polymerase chain reaction) amplified and sequenced to determine the presence of drug resistance mutations. In a similar fashion, E. coli cultures will also be used to determine the minimal inhibitory concentration (MIC) of these compounds to evaluate their ability to pass through the membrane barrier. Analysis of these compounds by these methods will allow us to evaluate and optimize chemical characteristics of the compounds before moving into an animal model and ensure the over all success of this project.

\section{Expanding the Pool of Known DHPS Structures}

With the assistance of the bioinformatics group at the Hartwell Center at St. Jude (Dr. Perdeep Mehta), we identified the DHPS gene from the genomic sequence of $F$. tularensis and discovered that it is fused with the gene for HPPK. Analysis of the sequence revealed that the C-terminal helix of DHPS, a key component of the TIM barrel, is missing. To ensure that the sequence is correct, we have obtained the genomic DNA and resequenced the DHPS region. Our initial sequence is correct and the gene has been PCR amplified and introduced into our standard pET28a expression vector, and pure soluble protein is now available for crystallization trials (Figure 6.1). However, biochemical data suggest that the DHPS component is inactive while the HPPK is active 
(data not shown). Further analysis into the cause of inactivity is necessary and will be a priority in this project as it is likely to reveal crucial information regarding the enzyme mechanism. Y. pestis genomic DNA was obtained from Dr. Brubaker (Michigan State University), the gene was PCR amplified, expressed, and the purified protein is currently undergoing crystallization trials (Figure 6.1). The Y. pestis gene is a standard bacterial enzyme and is fully functional (data not shown).

We will use crystallographic methods to incorporate other infectious agents into our inhibitor studies including those with already known structures (S. aureaus, $M$. tuberculosis, etc.) as well as class A agents of biowarfare, F. tularensis and Y. pestis. The structure determination of the $F$. tularensis and $Y$. pestis should be relatively straightforward. Preliminary crystals have been observed and are in the process of optimization. We anticipate that optimization of initial crystals will be successful. However, alternative methods are available should quality crystals prove difficult to obtain.

The reductive methylation of surface lysines has been shown to alter the crystal formation of various proteins and is a straightforward and efficient way for improving crystal quality (Rypniewski et al., 1993; Rayment, 1997; Kobayashi et al., 1999; Walter et al., 2006). In hen egg white lysozyme, it was shown that the methylation of all six lysine residues resulted in only minor changes in the overall protein architecture (Rypniewski et al., 1993). While several reductive methylation methods have been performed, we have chosen to employ formaldehyde as the alkylating reagent and dimethylamine borane complex as the reducing agent since they have been shown to have the mildest effects on the biochemical properties of the protein (Rayment, 1997). Examination of the B. anthracis DHPS structure revealed that the majority of lysines $(8 / 20)$ present in the structure were surface residues. Based on sequence alignment, we predict this to be true of the $Y$. pestis (9 lysines) and $F$. tularensis (41 lysines) DHPS.

Limited proteolysis as a second technique commonly used for obtaining quality crystals and is a straightforward technique. Using a variety of proteases, flexible regions of protein which may be involved in unfavorable interactions during crystallization can be identified and removed (Dong et al., 2007). Information obtained through treatment

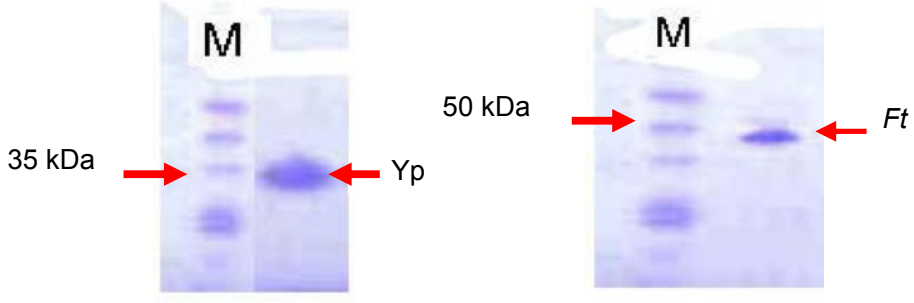

Figure 7.1: Purification Gels of $Y$. pestis and $F$. tularensis DHPS. Note that the $F$. tularensis DHPS has a larger molecular weight due to fusion with HPPK. 
with proteases can then be used to form new protein constructs for crystallization trials. This will be particularly useful with the $F$. tularensis construct as it may be necessary to cleave the HPPK and the DHPS enzymes to produce diffraction quality crystals.

As mentioned above, the $F$. tularensis DHPS was found to be inactive. This is unexpected as the active site residues are present in the sequence and align well with the DHPS sequence of other organisms. One notable difference in the $F$. tularensis sequence is the loss of the c-terminal helix which is an essential component of the TIM barrel. We will use site-directed mutagenesis to reinstate this terminal helix and study the effects on activity using the ELIPA. In addition, microbiology techniques will be used to determine the effects of the F. tularensis DHPS in E. coli cells. This will yield one of two results. First, $E$. coli cells are able to grow using the $F$. tularensis DHPS indicating that the enzyme requires additional elements not present in our assay. Second, E. coli cells are unable to grow indicating that the enzyme is nonfunctional. In the latter scenario, it will be necessary to examine the genomic sequence of $F$. tularensis to determine the presence of divergent orthologs which may explain this unusual result. Furthermore, Larrson et al. has evaluated the genome sequence of $F$. tularensis and identified several holes in a variety of metabolic pathways indicating that the bacterium is becoming increasingly host-dependent. While they do not indicate any holes in the folate pathway based on sequence analysis, this may explain the inactive form of DHPS. Examination of why this enzyme is inactive remains an important aim for this project and will undoubtedly provide crucial evidence into the enzymatic mechanism.

\section{Conclusions}

In an increasingly mobile and interconnected world, the transfer of infectious disease has become a serious threat through out the entire world and given rise to a need for novel therapeutics. The research proposed in this thesis gives a rational, valid method for overcoming the problems posed by present antibiotics and a plan for developing improved drug therapeutics. 


\section{List of References}

Achari, A. Somers, D.O., Champness, J.N., Bryant, P.K., Rosemond, J., and Stammers, D.K. (1997). Crystal structure of the anti-bacterial sulfonamide drug target dihydropteroate synthase. Nature Struct. Biol., 4:490-497.

Babaoglu, K., Page, M.A., Jones, V.C., McNeil, M.R., Dong, C., Naismith, J.H., Lee, R.E. (2003). Novel inhibitors of an emerging target in Mycobacterium tuberculosis; substituted thiazolidinones as inhibitors of dTDP-rhamnose synthesis. Bioorg. Med. Chem. Lett., 13:3227-3230.

Babaoglu, K., Qi, J., Lee, R.E. and White, S.W. (2004). Crystal structure of 7,8dihydropteroate synthase from Bacillus anthracis: mechanism and novel inhibitor design. Structure, 12 :1705-1717.

Baca, A.M., Sirawaraporn, R., Turley, S., Sirawaraporn, W., and Hol, W.G.J. (2000). Crystal structure of Mycobacterium tuberculosis 6-hydroxymethy-7,8dihydropteroate synthase in complex with pterin monophosphate: new insight into the enzymatic mechanism and sulfa-drug action. J. Mol. Biol., 302:1193-1212.

Bijvoet JM, Burgers WG, Hägg G, eds. (1972). Early Papers on Diffraction of X-rays by Crystals (Volume II). Utrecht: published for the International Union of Crystallography by A. Oosthoek's Uitgeversmaatschappij N.V..

Bragg WH (1907). The nature of Röntgen rays. Transactions of the Royal Society of Science of Australia 31: 94-98.

Bragg WH (1908). The nature of $\gamma$ - and X-rays. Nature 77: 270-271.

Bragg WH (1910). The consequences of the corpuscular hypothesis of the $\gamma$ - and X-rays, and the range of $\beta$-rays. Philosophical Magazine 20: 385-416.

Bragg WH (1912). On the direct or indirect nature of the ionization by X-rays. Philosophical Magazine 23: 647-650.

Bragg WL (1912). The Specular Reflexion of X-rays. Nature 90: 410.

Bragg WL (1913). The Diffraction of Short Electromagnetic Waves by a Crystal. Proceedings of the Cambridge Philosophical Society 17: 43-57.

Brown, G.M. (1962). The biosynthesis of folic acid. Inhibition by sulfonamides. J. Biol. Chem., 237:536-540. 
Brown, R., Joseph, M., Leigh, T. and Swain, M.L. (1977). Synthesis and reactions of 7,8dihydro-8-methylpterin and 9-methylguanine 7-oxide. J. Chem. Soc. Perkin Transactions, 1003-1009.

Cheng, Y. and Pursoff, W.H. (1973). Relationship between the inhibition constant $\mathrm{K}_{\mathrm{i}}$ and the concentration of inhibitor which causes 50 percent inhibition $\left(\mathrm{IC}_{50}\right)$ of an enzyme reaction. Biochem. Pharmocal., 22: 3099-3108.

Crowfoot Hodgkin D (1935). X-ray Single Crystal Photographs of Insulin. Nature 135: 591-59.

Dallas, W.S., K. Inderjit, and P. H. Ray (1992). Cloning, sequencing and enhanced expression of the dihydropteroate synthase gene of Escherichia coli MC4100. J. Bacteriol., 174: 5961-5970.

Domagk, G. (1935). Ein beitrag zur chemotherapie der bakteriellen infektionen. Dtsch med Wschr., 7: 250-253.

Dong, A., X. Xu, A.M. Edwards, and C. Chang. (2007). In situ proteolysis for protein crystallization and structure determination. Nature Methods., 4: 1019-1021.

Einstein, A (1905). Über einen die Erzeugung und Verwandlung des Lichtes betreffenden heuristischen Gesichtspunkt (trans. A Heuristic Model of the Creation and Transformation of Light). Annalen der Physik 17: 132-148.

Emsley P and Cowtan K. (2004). Coot: Model Building Tools for Molecular Graphics. Acta Crystallographica Section D-Biological Crystallography., 60: 2126-2132. Part $12 \mathrm{Sp}$. Iss.

Ewald PP, editor, and numerous crystallographers (1962). Fifty Years of X-ray

Diffraction. Utrecht: published for the International Union of Crystallography by A. Oosthoek's Uitgeversmaatschappij N.V..

Fermér, C., B.E. Kristiansen, O. Skold and G. Swedberg. (1995). Sulfonamide resistance in Neisseria meningitides as defined by site-directed mutagenesis could have its origin in other species. J. Bacteriol., 177: 4669-4675.

Fermér, C. and Swedberg, G. (1997). Adaptation to sulfonamide resistance in Neisseria meningitidis may have required compensatory changes to retain enzyme function: Kinetic analysis of dihydropteroate synthases from $N$. meningitidis expressed in a knockout mutant of Escherichia coli. J. Bacteriol., 179:831-837.

Gibreel, A. and Sköld, O. (1999). Sulfonamide resistance in clinical isolates of Campylobacter jejuni: mutational changes in the chromosomal dihydropteroate synthase. Antimcrob. Agents. Chemother., 43:2156-2160. 
Hampele, I.C., D’Arcy, A., Dale, G.E., Kostrewa, D., Nielsen, J., Oefner, C., Page, M.G.P., Schönfeld, H.-J., Stüber, D. and Then, R.L. (1997). Structure and function of the dihydropteroate synthase from Staphylococcus aureus. J. Mol. Biol., 268: 21 30 .

Irwin, J.J. and Shoichet, B.K. (2005). ZINC - a free database of commercially available compounds for virtual screening. .J. Chem. Inf. Model., 45: 177-182.

Kai, M., Matsuoka, M., Nakata, N., Maeda, S., Gidoh, M., Maeda, Y., Hashimoto, K., Kobayashi, K. and Kashiwabara, Y. (1999). Diaminodiphenylsulfone resistance of Mycobacterium leprae due to mutations in the dihydropteroate synthase gene. FEMS Microbiol. Letters, 177: 231-235.

Kasekarn, W., Sirawaraporn, R., Chahomchuen, T., Cowman, A.F. and Sirawaraporn, W. (2004). Molecular characterization of bifunctional hydroxymethyldihydropterin pyrophosphokinase-dihydropteroate synthase from Plasmodium falciparum. Mol. and Biochem. Parasitology, 137: 43-53.

Kazanjian, P., Locke, A. B., Hossler, P. A., Lane, B. R., Bartlett, M. S., Smith, J. W., Cannon, M. \& Meshnick, S. R. (1998). Pneumocystis carinii mutations associated with sulfa and sulfone prophylaxis failures in AIDS patients. AIDS, 12: 873-878.

Kepler, J. (1611). Strena seu de Nive Sexangula. Frankfurt: G. Tampach. http://www.thelatinlibrary.com/kepler/strena.html. 11/01/2008.

Kobayashi, M., M. Kubota, and Y. Matsuura. (1999). Crystallization and improvement of crystal quality for X-ray diffraction of maltooligosyl trehalose synthase by reductive methylation of lysine residues. Acta Cryst. D, 55: 931.

Lane, B. R., Ast, J. C., Hossler, P. A., Mindell, D. P., Bartlett, M. S., Smith, J. W. \& Meshnick, S. R. (1997). Dihydropteroate synthase polymorphisms in Pneumocystis carinii. J. Infect. Dis., 175: 482-485.

Lopez, P., Espinosa, M., Greenberg, B. \& Lacks, S. A. (1987). Sulfonamide resistance in Streptococcus pneumoniae: DNA sequence of the gene encoding dihydropteroate synthase and characterization of the enzyme. J. Bacteriol., 169: 4320-4326.

Maskell, J. P., Sefton, A. M. \& Hall, L. M. (1997). Mechanism of sulfonamide resistance in clinical isolate of Streptococcus pneumoniae. Antimicrob. Agents Chemother., 41: 2121-2126.

Mei, Q., Gurunathan, S., Masur, H. \& Kovacs, J. A. (1998). Failure of co-trimoxazole in Pneumocystis carinii infection and mutations in dihydropteroate synthase gene. Lancet, 351: 1631-1632. 
Larrson, Par, Petra Oyston, Patrick Chain, and May Chu. (2005). The Complete Genome Sequence of Francisella tularensis, the Causative Agent of Tularemia. Nature Genetics, 37: 153-159.

Lawrence, M.C., Iliades, P., Fernley, R.T., Berglez, J., Pilling, P.A. and Macreadie, I.G. (2005). The three-dimensional structure of the bifunctional 6-hydroxymethyl-7,8dihydropterin pyrophosphokinase/ dihydropteroate synthase of Saccharomyces cerevisiae. J. Mol. Biol., 348: 655-670.

Lever, O.W., Jr., Bell, L.N., McGuire, H.M. and Ferone, R. (1985). Monocyclic pteridine analogues. Inhibition of Escherichia coli dihydropteroate synthase by 6-amino-5nitrosoisocytosines. J. Med. Chem., 28: 1870-1874.

Lever, O.F. (1986). Inhibitors of dihydropteroate synthase: substituent effects in the sidechain aromatic ring of 6-[[3-(aryloxy0propyl]amino]-5-nitroisocytosines and synthasis and inhibitory potency of bridged 5-nitroisocytosine-p-aminobenzoic acid analogues. J. Med. Chem., 29: 665-670.

Levy, Colin, David Minnis, Jeremy P. Derrick. (2008). Dihydropteroate synthase from Streptococcus pneumoniae: Structure, ligand recognition and mechanism of sulfonamide resistance. Biochemical Journal. In progress.

Li, R., Sirawaraporn, R., Chitnumsub, P., Sirawaraporn, W., Wooden, J., Athappilly, F., Turley, S. and Hol, W. G. (2000). Three-dimensional structure of M. tuberculosis dihydrofolate reductase reveals opportunities for the design of novel tuberculosis drugs. J. Mol. Biol., 295: 307-323.

McCoy, A. J., R. W. Grosse-Kunstleve, P. D. Adams, M. D. Winn, L.C. Storoni and R.J. (2007). Phaser crystallographic software. J. Appl. Cryst., 40: 658-674.

Nopponpunth, V., Sirawaraporn, W., Greene, P.J. and Santi, D.V. (1999). Cloning and expression of Mycobacterium tuberculosis and Mycobacterium leprae dihydropteroate synthase in Escherichia coli. J. Bact., 181: 6814-6821.

Ochiai, K., Yamanaka, T Kimura K and Sawada, O (1959). Inheritance of drug resistance (and its tranfer) between Shigella strains and Between Shigella and E.coli strains. Hihon Iji Shimpor, 1861: 34.

Padaycachee, T. and Klugman, K. P. (1999). Novel expansions of the gene encoding dihydropteroate synthase in trimethoprim-sulfamethoxazole-resistant Streptococcus pneumoniae. Antimicrob. Agents Chemother., 43: 2225-2230.

Patel, O., Jacqueline Satchell, Jonathan Baell, Ross Fernley, Peter Coloe, and Ian Macreadie. (2003). Inhibition studies of sulfonamide-containing folate analogs in yeast. Microb. Drug. Resist., 9: 139-146. 
Rayment (1997). Reductive Alkylation of Lysine Residues to Alter Crystallization Properties of Proteins. Methods in Enzymology, 276: 171.

Rock, C.O., H.W. Park, and S. Jackowski. (2003). Role of Feedback Regulation of Pantothenate Kinase (CoA) in Control of Coenzyme A Levels in Escherichia coli. J. of Bact., 185: 3410-3415.

Roland, S. Ferone, R., Harvey, R.J., Styles, V.L. and Morrison, R.W. (1979). The characteristics and significance of sulfonamides as substrates for Escherichia coli dihydropteroate synthase. J. Biol. Chem., 254: 10337-10345.

Rypniewski, W.R., H.M. Holden, and I. Rayment. (1993). Structural consequences of reductive methylation of lysine residues in hen egg white lysozyme: an X-ray analysis at 1.8-A resolution. Biochemistry, 21: 9851.

Sköld, O. (2000). Sulfonamide resistance mechanisms and trends. Drug Resistance Updates, 3: 155-160.

Sköld, O. (2001). Resistance to trimethoprim and sulfonamides. Vet. Res., 32: 261-273.

Steno, N (1669). De solido intra solidum naturaliter contento dissertationis prodromus. Florentiae.

Swedberg, G., Ringertz, S. and Sköld, O. (1998). Sulfonamide resistance in Streptococcus pyogenes is associated with differences in amino acid sequence of its chromosomal dihydropteroate synthase. Antimicrob. Agents Chemother. 42: 10621067.

U.S. Holocaust Memorial Museum. Trials of War Criminals before the Nuremberg Military Tribunals under Control Council Law No. 10. Nuremberg, October 1946 April 1949. Washington D.C.: U.S. G.P.O, 1949-1953.

Valderas M.W. (2008). Examination of intrinsic sulfonamide resistance in Bacillus anthracis: A novel assay for dihydropteroate synthase. Biochim. Biophys. Acta., 5: 848-853.

Vedantam, G., Guay, G. G., Austria, N. E., Doktor, S. Z. \& Nichols, B. P. (1998). Characterization of mutations contributing to sulfathiazole resistance in Escherichia coli. Antimicrob. Agents Chemother., 42: 88-93.

Vinnicombe, H.G. and Derrick, J.P. (1999). Dihydropteroate synthase from Streptococcus pneumoniae: Characterization of substrate binding order and sulfonamide inhibition. B.B.R.C. 258: 752-757.

Walter, T.S., C Meier, R. Assenberg, K.F. Au, J. Ren. (2006). Lysine Methylation as a Routine Rescue Strategy for Protein Crystallization. Structure, 14: 1617. 
Wang, P., Lee, C.-S., Bayoumi, R., Djimde, A., Doumbo, O., Swedberg, G., Dao, L.D., Mshinda, H., Tanner, M., Watkins, W.M., Sims, P.F.G. and Hyde, J.E. (1997).

Resistance to anitfolates in Plasmodium falciparum monitored by sequence analysis of dihydropteroate synthase and dihyrofolate reductase alleles in a large number of field samples of diverse origins. Mol. Biochem. Paristol., 89: 161-177.

Zheng, Yong-Mei and C.O. Rock (2004). Acyl Carrier Proteins Is a Cellular Target for the Antibacterial Action of the Pantothenamide Class of Pantothenate Antimetabolites. J. of Biol. Chem., 279: 50969-50975. 


\section{Vita}

Katherine A. Ayers was born in Arkadelphia, Arkansas, on March 10, 1983. She received a dual Bachelor of Science degree in Chemistry and Theology from Union University in Jackson, Tennessee, in May 2005. She joined the Integrated Program of Biological Sciences majoring in Biochemistry at the University of Tennessee Health Science Center in Memphis, Tennessee, in August 2005 and received her Master of Science degree with a major in Cell Biology and Biochemistry in May 2009. 